\title{
High-energy gamma-ray and neutrino backgrounds from clusters of galaxies and radio constraints
}

\author{
Fabio Zandanel $^{1}$, Irene Tamborra ${ }^{1}$, Stefano Gabici $^{2}$, and Shin'ichiro Ando ${ }^{1}$
}

\author{
1 GRAPPA Institute, University of Amsterdam, Science Park 904, 1098 XH Amsterdam, The Netherlands \\ e-mail: f.zandanel@uva.nl \\ 2 APC, Université Paris Diderot, CNRS/IN2P3, CEA/Irfu, Observatoire de Paris, Sorbonne Paris Cité, 75014 Paris, France
}

Received 31 October 2014 / Accepted 10 March 2015

\section{ABSTRACT}

\begin{abstract}
Cosmic-ray protons accumulate for cosmological times in clusters of galaxies because their typical radiative and diffusive escape times are longer than the Hubble time. Their hadronic interactions with protons of the intra-cluster medium generate secondary electrons, gamma rays, and neutrinos. In light of the high-energy neutrino events recently discovered by the IceCube neutrino observatory, for which galaxy clusters have been suggested as possible sources, and the forthcoming results from the Fermi gamma-ray survey, we here estimate the contribution from galaxy clusters to the diffuse gamma-ray and neutrino backgrounds. We modelled the cluster population by means of their mass function, using a phenomenological luminosity-mass relation applied to all clusters, as well as a detailed semi-analytical model. In the latter model, we divide clusters into cool-core/non-cool-core, and loud/quiet subsamples, as suggested by observations, and model the cosmic-ray proton population according to state-of-the-art hydrodynamic numerical simulations. Additionally, we consider observationally-motivated values for the cluster magnetic field. This is a crucial parameter since the observed radio counts of clusters need to be respected owing to synchrotron emission by secondary electrons. For a choice of parameters respecting current constraints from radio to gamma rays, and assuming a proton spectral index of -2 , we find that hadronic interactions in clusters contribute less than $10 \%$ to the IceCube flux and much less to the total extragalactic gamma-ray background observed by Fermi. They account for less than $1 \%$ for spectral indices $\leq-2$. The high-energy neutrino flux observed by IceCube can be reproduced without violating radio constraints only if a very hard (and speculative) spectral index $>-2$ is adopted. However, this scenario is in tension with the high-energy IceCube data, which seems to suggest a spectral energy distribution of the neutrino flux that decreases with the particle energy. We prove that IceCube should be able to test our most optimistic scenarios for spectral indices $\geq-2.2$ by stacking a few nearby massive galaxy clusters. In the case of proton-photon interactions in clusters, we find that very likely protons do not reach sufficiently high energies to produce neutrinos in these environments. We argue that our results are optimistic because of our assumptions and that clusters of galaxies cannot make any relevant contribution to the extragalactic gamma-ray and neutrino backgrounds in any realistic scenario. Finally, we find that the cluster contribution to the angular fluctuations in the gamma-ray background is subdominant, less than $10 \%$ on sub-degree scales.
\end{abstract}

Key words. galaxies: clusters: general - gamma rays: diffuse background - gamma rays: galaxies: clusters - neutrinos

\section{Introduction}

The extragalactic gamma-ray background (EGB) is the measured radiation that remains after subtracting all known sources from the observed gamma-ray sky. The EGB was measured by the SAS-2 satellite for the first time (Fichtel et al. 1977) then by EGRET (Sreekumar et al. 1998; Strong et al. 2004) and the Fermi-Large Area Telescope (LAT; Fermi LAT Collaboration $2010 b$, 2015a) most recently. The EGB is likely due to the sum of contributions from different unresolved sources, such as active galactic nuclei (AGN), star-forming galaxies, pulsars, gamma-ray bursts, and intergalactic shocks produced by structure formation (see, e.g., Dermer 2007; Abdo et al. 2010; Stecker \& Venters 2011; Siegal-Gaskins et al. 2011; Ackermann et al. 2012; Fornasa et al. 2013; Di Mauro et al. 2014a,b; Tamborra et al. 2014; Ajello et al. 2015; Di Mauro \& Donato 2015, and references therein).

Recently, the IceCube neutrino observatory at the South Pole has reported evidence of extraterrestrial neutrinos (Aartsen et al. 2013, 2014a). The four-year IceCube dataset consists of 37 events that exceed the atmospheric background with a significance of more than $5 \sigma$ (Aartsen et al. 2014a). The neutrino data are compatible with a flux isotropically distributed in the sky, with astrophysical origin and with a possible cutoff at a few PeV. The origin of these events is unknown (see Waxman 2013; and Anchordoqui et al. 2014a, for recent reviews; see also Winter 2014). However, the isotropic distribution in the sky of the observed events suggests that they might come from various extragalactic $\sim 100 \mathrm{PeV}$ cosmic-ray (CR) accelerators, such as gamma-ray bursts, especially untriggered ones (Waxman \& Bahcall 1997; Hümmer et al. 2012; Murase \& Ioka 2013; Liu \& Wang 2013); AGN (Waxman \& Bahcall 1999; Halzen \& Hooper 2005; Stecker 2013; Winter 2013; Murase et al. 2014; Becker Tjus et al. 2014); star-forming galaxies including starbursts, galaxy mergers, and AGN (Loeb \& Waxman 2006; Tamborra et al. 2014; Lacki et al. 2011; Murase et al. 2013; He et al. 2013; Liu et al. 2014; Katz et al. 2013; Kashiyama \& Meszaros 2014; Anchordoqui et al. 2014c; Chang \& Wang 2014; Tavecchio \& Ghisellini 2014); intergalactic shocks and active galaxies embedded in structured regions (Murase et al. 2013); and hypernovae and supernova remnants (Chakraborty \& Izaguirre 2015; Senno et al. 2015). A galactic origin for the neutrinos has also been proposed (Ahlers \& Murase 2014; Fox et al. 2013; Joshi et al. 2014; Taylor et al. 2014; Anchordoqui et al. 2014b), 
as well as mixed scenarios of galactic and extragalactic neutrino sources (Ahlers \& Murase 2014; Razzaque 2013; Fox et al. 2013; Joshi et al. 2014; Murase et al. 2014; Padovani \& Resconi 2014). Exotic models including PeV dark matter decay scenarios have been discussed, too (Feldstein et al. 2013; Esmaili \& Serpico 2013; Esmaili et al. 2014).

As shown in Murase et al. (2013), a multi-messenger connection between the measured neutrino fluxes and their photon counterparts could be crucial for unveiling the origin of the high-energy neutrinos, regardless of the physics of their sources. In the following, we assume that the IceCube high-energy neutrinos have an extragalactic origin and are produced in protonproton collisions. In such a scenario we would expect sources to also emit gamma rays at a flux comparable to that of neutrinos (see, e.g., Kelner et al. 2006); however, the neutrinos could also be produced in proton-photon interactions (see, e.g., Kelner \& Aharonian 2008).

Clusters of galaxies are the latest and largest structures to form in the Universe. During their assembly, energies of the same order of magnitude as the gravitational binding energy, $10^{61}-10^{63} \mathrm{erg}$, should be dissipated through structure-formation shocks and turbulence (Voit 2005). Therefore, even if only a small part of this energy goes into particle acceleration, clusters should host significant non-thermal emission from radio to gamma rays (see, e.g., Brunetti \& Jones 2014).

The contribution of clusters of galaxies to the EGB has been discussed by several authors (Loeb \& Waxman 2000; Keshet et al. 2003; Gabici \& Blasi 2003; Ando \& Nagai 2008; Zandanel $\&$ Ando 2014). It has been argued that CR hadronic interactions in galaxy clusters could be responsible for a neutrino flux that is comparable to the one recently observed by IceCube (Murase et al. 2008, 2013; Kotera et al. 2009; Murase \& Beacom 2013). However, such hadronic interactions could have a dramatic impact on the radio frequencies since secondary electrons are also produced in proton-proton interactions and radiate synchrotron emission when interacting with the magnetic fields in clusters of galaxies. The radio emission from secondary electrons needs to respect radio counts of galaxy clusters (Giovannini et al. 1999; Venturi et al. 2007, 2008; Kale et al. 2013), since the cluster diffuse synchrotron radio emission has been observed (see, e.g., Feretti et al. 2012).

In this work, we estimate the possible contribution to the extragalactic gamma-ray and neutrino backgrounds from galaxy clusters assuming that gamma rays and neutrinos mainly originate in proton-proton interactions, while for the first time taking the consequences in the radio regime into account. We compare our model estimates to the isotropic diffuse gamma-ray background measured by Fermi (Fermi LAT Collaboration 2015a) and to the neutrino flux measured by IceCube (Aartsen et al. 2014a). We also discuss the small-scale anisotropies in EGB recently detected with Fermi (Fermi LAT Collaboration 2012) and compare the measurements with cluster models.

This paper is organised as follows. In Sect. 2, we briefly discuss proton-proton interactions in galaxy clusters and explain how we calculate the emission from secondary electrons, photons, and neutrinos. We then introduce the mass function of galaxy clusters and a phenomenological luminosity-mass relation in Sect. 3. In Sect. 4, we refine our approach by using a detailed semi-analytical model based on state-of-the-art numerical simulations of CRs in clusters and test the robustness of our results with respect to the adopted parameters. We compare our results with stacking upper limits by the IceCube telescope and discuss future detection prospects in Sect. 5. We briefly discuss the neutrino contribution from proton-photon interactions in clusters in Sect. 6 and the angular power spectrum of the EGB in Sect. 7. Finally, in Sect. 8, we summarise our findings.

\section{Secondaries from proton-proton interactions}

The CR protons accumulate in galaxy clusters for cosmological times (Völk et al. 1996; Berezinsky et al. 1997) and interact with the thermal protons of the intra-cluster medium (ICM) generating secondary particles: electrons, neutrinos, and highenergy photons (Dennison 1980; Blasi \& Colafrancesco 1999; Miniati et al. 2001; Pfrommer \& Enßlin 2004; Blasi et al. 2007; Pfrommer et al. 2008; Kushnir \& Waxman 2009; Kotera et al. 2009; Pinzke \& Pfrommer 2010). While the ICM density is typically well known from X-ray measurements of its bremsstrahlung emission, the CR proton spectral and spatial distributions in galaxy clusters are unknown. In fact, whereas the diffuse radio emission observed in several clusters proves the presence of relativistic electrons, direct proof of proton acceleration has yet to be found.

Gamma-ray observations of the possible hadronic-induced emission started to put tight constraints on the proton content of clusters (H.E.S.S. Collaboration 2009a,b; MAGIC Collaboration 2010, 2012; Fermi LAT Collaboration 2010a, 2014; VERITAS Collaboration 2012; Ando \& Nagai 2012; Huber et al. 2013; Vazza \& Brüggen 2014; Zandanel \& Ando 2014; Prokhorov \& Churazov 2014; Griffin et al. 2014). Gamma-ray limits also suggest that secondary electrons cannot be uniquely responsible for the observed radio emission in galaxy clusters, at least in the case of the so-called giant radio haloes found in merging clusters like Coma (Brunetti et al. 2012; Zandanel et al. 2014b). As we discuss in the following, an important implication for our purposes is that the observed radio counts represent an optimistic upper limit for the radio emission from secondary electrons since only a fraction of it can have a hadronic origin.

Assuming a power law in momentum for the spectral distribution of CR protons in clusters, $f(p) \mathrm{d} p=\rho_{\mathrm{CR}} p^{-\alpha_{\mathrm{p}}} \mathrm{d} p$, the radio synchrotron luminosity of secondary electrons at a frequency $f$ can be expressed as (adapted from Pfrommer et al. 2008)

$L_{f}=A_{f} \int \rho_{\mathrm{CR}} \rho_{\mathrm{ICM}} \frac{\epsilon_{B}}{\epsilon_{B}+\epsilon_{\mathrm{CMB}}}\left(\frac{\epsilon_{B}}{\epsilon_{B_{\mathrm{c}}}}\right)^{\frac{\alpha_{\mathrm{p}-2}}{4}} \mathrm{~d} V$,

where $\rho_{\mathrm{CR}}$ and $\rho_{\mathrm{ICM}}$ are the CR proton and ICM density distributions, respectively, while $\epsilon_{B}=B^{2} / 8 \pi$ and $\epsilon_{\mathrm{CMB}}$ are the energy densities of the cluster magnetic fields and the cosmic microwave background $(\mathrm{CMB})^{1}$. The parameter $\epsilon_{B_{\mathrm{c}}}$ is the magnetic energy density corresponding to a characteristic magnetic field $B_{\mathrm{c}}=31(v / \mathrm{GHz}) \mu \mathrm{G}$ for the synchrotron mechanism, and $A_{f}$ encloses the spectral information (Pfrommer et al. 2008). The gamma-ray luminosity of secondary photons at an energy $E$ is defined as

$L_{\gamma}=A_{\gamma} \int \rho_{\mathrm{CR}} \rho_{\mathrm{ICM}} \mathrm{d} V$,

with $A_{\gamma}$ enclosing the spectral information (Pfrommer et al. 2008).

In the following, we make use of Eqs. (1) and (2) to calculate the hadronic-induced emission in galaxy clusters at radio and gamma-ray frequencies. The spectral multipliers $A_{f}$ and $A_{\gamma}$ were

\footnotetext{
The total energy density of photons should also include the contribution from star light: $\epsilon_{\mathrm{ph}}=\epsilon_{\mathrm{stars}}+\epsilon_{\mathrm{CMB}}$. However, $\epsilon_{\mathrm{stars}}$ is subdominant in the cluster volume (see, e.g., Fig. 5 of Pinzke et al. 2011), therefore $\epsilon_{\mathrm{ph}} \approx \epsilon_{\mathrm{CMB}}$.
} 
obtained in Pfrommer \& Enßlin (2004) as analytical approximations of full proton-proton interaction simulations. The analytical expressions for $A_{f}$ and $A_{\gamma}$ reproduce the results of numerical simulations from energies around the pion bump ( 100 MeV) up to a few hundred $\mathrm{GeV}$. A more precise formalism has been derived by Kelner et al. (2006) for the TeV-PeV energy range, relevant to calculating the neutrino fluxes. Therefore, we correct the gamma-ray spectra obtained by adopting the analytical approximations with the recipe in Kelner et al. (2006) for energies above $\sim 0.1-1 \mathrm{TeV}$. The transition energy between the two approximations depends on $\alpha_{\mathrm{p}}$, and it was chosen as the energy at which the two models coincide.

We compute the corresponding neutrino spectra as prescribed in Kelner et al. (2006). When assuming that protonproton interactions are the main interactions producing neutrinos and gamma rays, the neutrino intensity for all flavours could also be approximately obtained as a function of the gammaray flux (Ahlers \& Murase 2014; Anchordoqui et al. 2004): $L_{v}\left(E_{v}\right) \approx 6 L_{\gamma}\left(E_{\gamma}\right)$, with $E_{v} \approx E_{\gamma} / 2$, where we ignored the absorption during the propagation of gamma rays for simplicity. From this approximation, one finds that, at a given energy, $L_{v} / L_{\gamma} \sim 1.5$ for $\alpha_{\mathrm{p}}=2$. However, detailed calculations by Berezinsky et al. (1997) and Kelner et al. (2006) show that this ratio is slightly smaller for spectral indices $\alpha_{\mathrm{p}}>2$ and slightly higher for $\alpha_{\mathrm{p}}<2$.

We do not assume any CR spectral cut-off at high energies or any spectral steepening due to the high-energy protons that are no longer confined to the cluster (Völk et al. 1996; Berezinsky et al. 1997; Pinzke \& Pfrommer 2010), and thus, as discussed in the following, our results should be considered as conservative. While this is not relevant when comparing with the Fermi data, it might be relevant for the high-energy neutrino flux.

Since the larger contribution to the total diffuse intensity comes from nearby galaxy clusters (see Fig. 5 and comments therein), we additionally omit the absorption of high-energy gamma rays owing to interactions with the extragalactic background light because this becomes relevant only at high redshifts (see, e.g., Domínguez et al. 2011). We note that our conclusions do not change even when relaxing any of the above approximations.

\section{Phenomenological luminosity-mass relation}

In this section, we estimate the maximum possible contribution to the extragalactic gamma-ray and neutrino backgrounds from hadronic interactions in galaxy clusters using a simplified phenomenological approach for the luminosity-mass relation.

\subsection{Modelling the diffuse gamma-ray intensity}

The total gamma-ray intensity from all galaxy clusters in the Universe at a given energy $(\mathrm{d} N / \mathrm{d} A \mathrm{~d} t \mathrm{~d} E)$ is

$$
\begin{aligned}
I_{\gamma}=\int_{z_{1}}^{z_{2}} \int_{M_{500, \lim }} & \frac{L_{\gamma}\left(M_{500}, z\right)(1+z)^{2}}{4 \pi D_{\mathrm{L}}(z)^{2}} \\
& \times \frac{\mathrm{d}^{2} n\left(M_{500}, z\right)}{\mathrm{d} V_{\mathrm{c}} \mathrm{d} M_{500}} \frac{\mathrm{d} V_{\mathrm{c}}}{\mathrm{d} z} \mathrm{~d} z \mathrm{~d} M_{500},
\end{aligned}
$$

where the cluster mass $M_{\Delta}$ is defined with respect to a density that is $\Delta=500$ times the critical density of the Universe at redshift $z$. Here, $V_{\mathrm{c}}$ is the comoving volume, $D_{\mathrm{L}}(z)$ the luminosity distance, and $\mathrm{d}^{2} n\left(M_{500}, z\right) / \mathrm{d} V_{\mathrm{c}} \mathrm{d} M_{500}$ is the cluster mass function for which we make use of the Tinker et al. (2008)

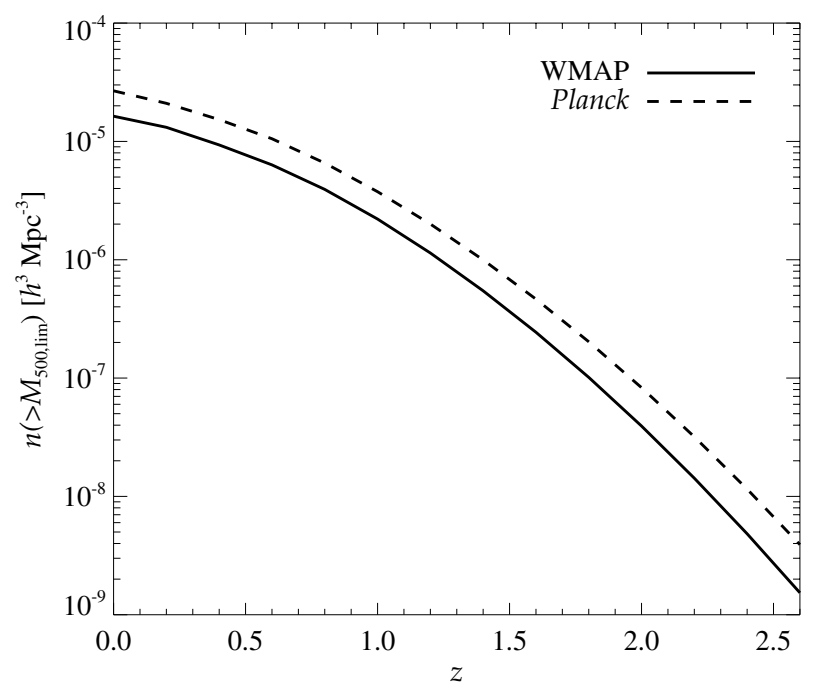

Fig. 1. Total number density of galaxy clusters for masses above $M_{500, \lim }=10^{13.8} h^{-1} M_{\odot}$ as a function of redshift. We show the number density obtained assuming the WMAP (Komatsu et al. 2011), our standard choice if not otherwise specified, and the Planck (Planck Collaboration XVI 2014) cosmological data. At redshift $z=2$, the number density is already negligible with respect to the lowest redshift.

formalism and the Murray et al. (2013) on-line application. The lower limit of the mass integration has been chosen to be $M_{500, \lim }=10^{13.8} h^{-1} M_{\odot}$, to account for large galaxy groups. The redshift integration goes from $z_{1}=0.01$, where the closest galaxy clusters are located, up to $z_{2}=2$. Where not otherwise specified, we assume $\Omega_{\mathrm{m}}=0.27, \Omega_{\Lambda}=0.73$, and the Hubble parameter $H_{0}=100 h_{70} \mathrm{~km} \mathrm{~s}^{-1} \mathrm{Mpc}^{-1}$ with $h_{70}=0.7$. Where we explicitly use $h$ in the units, as for $M_{500, \lim }$, we assume $H_{0}=100 h \mathrm{~km} \mathrm{~s}^{-1} \mathrm{Mpc}^{-1}$ with $h=1$. As shown in Fig. 1 (and discussed in Sects. 3.3 and 4.3), our conclusions are not affected by the specific choice of $z_{2}$ and $M_{500 \text {, lim }}$.

We calculate the total number of detectable galaxy clusters at $f=1.4 \mathrm{GHz}$, above the flux $F_{\min }$, as

$N_{1.4}\left(>F_{\text {min }}\right)=\int_{z_{1}}^{z_{2}} \int_{F_{\min }}^{\infty} \frac{\mathrm{d}^{2} n\left(F_{1.4}, z\right)}{\mathrm{d} V_{\mathrm{c}} \mathrm{d} F_{1.4}} \frac{\mathrm{d} V_{\mathrm{c}}}{\mathrm{d} z} \mathrm{~d} z \mathrm{~d} F_{1.4}$,

where $F_{1.4}=L_{1.4}(1+z) / 4 \pi D_{\mathrm{L}}(z)^{2}$, and we compare it with the radio counts from the National Radio Astronomy Observatory Very Large Array sky survey (NVSS) of Giovannini et al. $(1999)^{2}$. The flux $F_{\min }$ is defined as in Eq. (9) of Cassano et al. (2012) by adopting a noise-level multiplier $\xi_{1}=1$, which is appropriate, while slightly optimistic, for the low redshifts of the NVSS survey $(0.44 \leq z \leq 0.2)$, and a typical radio half-light radius of $R_{500} / 4$ (Zandanel et al. 2014b).

The function $\mathrm{d}^{2} n\left(F_{1.4}, z\right) / \mathrm{d} V_{\mathrm{c}} \mathrm{d} F_{1.4}$ is obtained numerically from $\mathrm{d}^{2} n\left(M_{500}, z\right) / \mathrm{d} V_{\mathrm{c}} \mathrm{d} M_{500}$ by calculating $L_{1.4}\left(M_{500}\right)$ from $L_{\gamma}\left(M_{500}\right)$ as explained in the following. We introduce a phenomenologically-driven gamma-ray luminosity-mass relation:

$\log _{10}\left[\frac{L_{\gamma}(100 \mathrm{MeV})}{\mathrm{s}^{-1} \mathrm{GeV}^{-1}}\right]=P_{1}+P_{2} \log _{10}\left(\frac{M_{500}}{M_{\odot}}\right)$,

2 We use the cumulative number density function as in Cassano et al. (2010). Cassano et al. (2010) do not use the fluxes of Giovannini et al. (1999), but rather the ones from follow-up observations of the same sample of galaxy clusters, which are higher than the NVSS ones (Cassano, priv. comm.). 
Table 1. Tested parameters and total gamma-ray and neutrino fluxes for the phenomenological luminosity-mass relation.

\begin{tabular}{|c|c|c|c|c|c|c|c|c|}
\hline$\alpha_{\mathrm{p}}$ & Loud [\%] & $B[\mu \mathrm{G}]$ & $P_{1}$ & Coma $(>100 \mathrm{MeV})$ & Perseus (>1 TeV) & $I_{\gamma}(100 \mathrm{MeV})$ & $I_{v}(250 \mathrm{TeV})$ & Notes \\
\hline 1.5 & 100 & $\begin{array}{c}\gg B_{\mathrm{CMB}} \\
1 \\
0.5 \\
\end{array}$ & $\begin{array}{l}18.60(18.35) \\
19.41(18.35) \\
19.91(18.35) \\
\end{array}$ & $\begin{array}{l}1.6(0.92) \times 10^{-11} \\
1.1(0.09) \times 10^{-10} \\
3.3(0.09) \times 10^{-10}\end{array}$ & $\begin{array}{l}1.7(0.92) \times 10^{-13} \\
1.1(0.09) \times 10^{-12} \\
3.4(0.09) \times 10^{-12}\end{array}$ & $\begin{array}{l}3.8(2.1) \times 10^{-10} \\
2.5(0.2) \times 10^{-9} \\
7.8(0.2) \times 10^{-9} \\
\end{array}$ & $\begin{array}{l}7.3(4.2) \times 10^{-19} \\
4.7(0.4) \times 10^{-18} \\
1.5(0.04) \times 10^{-17}\end{array}$ & $\begin{array}{l}\mathrm{N} \\
\mathrm{N} \\
\mathrm{N}\end{array}$ \\
\hline 2 & 100 & $\begin{array}{c}\gg B_{\mathrm{CMB}} \\
1 \\
0.5 \\
\gg B_{\mathrm{CMB}} \\
1 \\
0.5 \\
\end{array}$ & $\begin{array}{c}19.42 \\
20.65 \\
21.23(21.09) \\
19.60 \\
20.82 \\
21.40(21.09) \\
\end{array}$ & $\begin{array}{c}6.0 \times 10^{-11} \\
1.0 \times 10^{-9} \\
3.9(2.8) \times 10^{-9} \\
9.1 \times 10^{-11} \\
1.5 \times 10^{-9} \\
5.7(2.8) \times 10^{-9} \\
\end{array}$ & $\begin{array}{c}1.1 \times 10^{-14} \\
1.8 \times 10^{-13} \\
6.9(5.0) \times 10^{-13} \\
1.6 \times 10^{-14} \\
2.7 \times 10^{-13} \\
1.0(0.5) \times 10^{-12} \\
\end{array}$ & $\begin{array}{c}2.5 \times 10^{-9} \\
4.3 \times 10^{-8} \\
1.6(1.2) \times 10^{-7} \\
1.4 \times 10^{-9} \\
2.3 \times 10^{-8} \\
8.9(4.4) \times 10^{-8} \\
\end{array}$ & $\begin{array}{c}4.7 \times 10^{-21} \\
8.1 \times 10^{-20} \\
3.1(2.2) \times 10^{-19} \\
2.8 \times 10^{-21} \\
4.6 \times 10^{-20} \\
1.8(0.9) \times 10^{-19}\end{array}$ & G \\
\hline 2.2 & 100 & $\begin{array}{c}\gg B_{\mathrm{CMB}} \\
1 \\
0.5 \\
\end{array}$ & $\begin{array}{c}19.71 \\
21.10 \\
21.71(21.16) \\
\end{array}$ & $\begin{array}{c}1.0 \times 10^{-10} \\
2.6 \times 10^{-9} \\
1.0(0.3) \times 10^{-8} \\
\end{array}$ & $\begin{array}{c}3.6 \times 10^{-15} \\
8.7 \times 10^{-14} \\
3.6(1.0) \times 10^{-13} \\
\end{array}$ & $\begin{array}{c}4.9 \times 10^{-9} \\
1.2 \times 10^{-7} \\
4.9(1.4) \times 10^{-7} \\
\end{array}$ & $\begin{array}{c}5.9 \times 10^{-22} \\
1.4 \times 10^{-20} \\
5.9(1.7) \times 10^{-20} \\
\end{array}$ & $\mathrm{G}$ \\
\hline 2.4 & 100 & $\begin{array}{c}\gg B_{\mathrm{CMB}} \\
1 \\
0.5\end{array}$ & $\begin{array}{c}19.98 \\
21.54(21.21) \\
22.18(21.21)\end{array}$ & $\begin{array}{c}1.6 \times 10^{-10} \\
5.9(2.8) \times 10^{-9} \\
2.6(0.3) \times 10^{-8}\end{array}$ & $\begin{array}{c}1.2 \times 10^{-15} \\
4.2(1.9) \times 10^{-14} \\
1.8(0.2) \times 10^{-13}\end{array}$ & $\begin{array}{c}9.1 \times 10^{-9} \\
3.3(1.6) \times 10^{-7} \\
1.4(0.2) \times 10^{-6}\end{array}$ & $\begin{array}{c}7.0 \times 10^{-23} \\
2.5(1.2) \times 10^{-21} \\
1.1(0.1) \times 10^{-20}\end{array}$ & $\begin{array}{l}\mathrm{G} \\
\mathrm{G}\end{array}$ \\
\hline
\end{tabular}

Notes. For each $\alpha_{\mathrm{p}}$ and magnetic field, the $P_{1}$ parameter of the $L_{\gamma}(100 \mathrm{MeV})-M_{500}$, obtained by taking the NVSS radio counts into account, is reported in the fourth column. Columns 5 and 6: corresponding Coma-like and Perseus-like gamma-ray flux in $\mathrm{cm}^{-2} \mathrm{~s}^{-1}$, respectively, integrated above $100 \mathrm{MeV}$ and $1 \mathrm{TeV}$, and assuming the clusters $M_{500}$ as in Reiprich \& Böhringer (2002). Columns 7 and 8: total gamma-ray and neutrino (all flavours) intensity at $100 \mathrm{MeV}$ and $250 \mathrm{TeV}$, respectively, in $\mathrm{cm}^{-2} \mathrm{~s}^{-1} \mathrm{GeV}^{-1} \mathrm{sr}^{-1}$ for all the galaxy clusters in the Universe. Last column: "G" and "N", cases overshooting present gamma-ray and neutrino constraints, respectively. For $\alpha_{\mathrm{p}} \geq 2$, we report in parenthesis the values that respect the gamma-ray upper limit on Coma, while for $\alpha_{\mathrm{p}}=1.5$ we report in parenthesis the values matching the IceCube neutrino data averaging in the corresponding energy range.

where we omit the possible redshift-dependence for simplicity ${ }^{3}$. The radio luminosity can be obtained from the gamma-ray one by Eqs. (1) and (2).

In this section we assume that the magnetic field is independent of the radius in the radio-emitting region. Therefore, the relation between radio and gamma-ray luminosities becomes

$\frac{L_{\gamma}}{L_{f}}=\frac{A_{\gamma}}{A_{f}} \frac{\epsilon_{B}+\epsilon_{\mathrm{CMB}}}{\epsilon_{B}}\left(\frac{\epsilon_{B_{\mathrm{c}}}}{\epsilon_{B}}\right)^{\frac{\alpha_{\mathrm{p}-2}}{4}}$.

A special limit can be obtained for $B \gg B_{\mathrm{CMB}}$ in all the radioemitting region. In this case, under the hypothesis that electrons lose all their energy through synchrotron emission and $\alpha_{\mathrm{p}} \approx 2$, the relation between radio and gamma-ray luminosities becomes (Pfrommer 2008):

$$
\frac{L_{\gamma}}{L_{f}} \approx \frac{A_{\gamma}}{A_{f}} .
$$

Concerning the choice of the parameters in Eq. (5), we need to consider that $P_{1}, P_{2}, \alpha_{\mathrm{p}}, B$, and the fraction of loud clusters are degenerate when one tries to find the maximum allowed hadronic-induced emission. The concept of loud fraction comes from the fact that, even if clusters have the same X-ray luminosity and therefore the same mass, some of them host radio emission, but others do not show any sign of it with upper limits about an order of magnitude below the loud state. This is known as the radio-X-ray bimodality (Brunetti et al. 2009; Cassano et al. 2013). The most recent estimates suggest that the radio-loud percentage is about 20-30\% (Kale et al. 2013). The subdivision

\footnotetext{
3 Because the larger contribution to both the number of detectable clusters in radio (Zandanel et al. 2014b) and the total gamma-ray and neutrino fluxes is dominated by nearby clusters, the high-redshift dependence is negligible for our purposes (see Sects. 3.3 and 4.3 for more details).
}

of the cluster population into radio-loud and radio-quiet clusters is also reflected in the corresponding gamma-ray and neutrino fluxes. Therefore, from now on we refer to the two populations as "loud" and "quiet."

In this section we mainly consider the overly optimistic case where all the clusters are loud (100\% loud), while we show the case of $30 \%$ loud clusters for only one choice of $\alpha_{\mathrm{p}}$. In the following, to reduce the number of free parameters, we fix $P_{2}=$ $5 / 3 \simeq 1.67$; i.e., we assume that the hadronic-induced luminosity scales as the cluster thermal energy $E_{\text {th }} \propto M^{2} / R_{\text {vir }} \propto M^{5 / 3}$ (see also Sect. 3.3), where $R_{\text {vir }}$ is the virial radius. The chosen $P_{2}$ parameter roughly corresponds to what is found using the Zandanel et al. (2014a) multi-frequency mock cluster catalogue (MultiDark database; Riebe et al. 2013) for $L_{\gamma}(100 \mathrm{MeV})-M_{500}$, which typically lies in the range $\sim 1.5-1.65$ for different redshifts and different cluster populations (loud, quiet, cool-core, non-cool-core). The parameter $P_{1}$ is set free to vary under the constraint that it should respect the radio counts from the NVSS survey and current gamma-ray upper limits. We note that, once the thermal content of a cluster is known, the parameter $P_{1}$ could be seen as the efficiency of how much energy goes into $\mathrm{CR}$ acceleration.

We considered the Coma and Perseus cases for comparison with current gamma-ray upper limits on individual galaxy clusters. We took the Coma upper limit obtained from five years of Fermi data by Zandanel \& Ando (2014) as reference. We adopted their result for the disk model, a uniform filling of the cluster up to $R_{200}$, which is $F_{\mathrm{UL}}(>100 \mathrm{MeV})=$ $2.9 \times 10^{-9} \mathrm{~cm}^{-2} \mathrm{~s}^{-1}$, obtained for a spectral index of 2 . For Perseus, we assumed the upper limit obtained by the MAGIC Collaboration (2012) for the inner region of 0.15 as reference, which is $F_{\mathrm{UL}}(>1 \mathrm{TeV})=1.4 \times 10^{-13} \mathrm{~cm}^{-2} \mathrm{~s}^{-1}$, obtained for a spectral index of 2.2. We refer the reader to, for example, Table 1 of Huber et al. (2013) and Table 2 of MAGIC Collaboration (2010) for hints to how much the gamma-ray upper limits change 
when modifying the spectral index. Such a change is quantifiable within a factor of about two, which does not affect our conclusions, as we discuss later.

\subsection{Results: gamma-ray and neutrino backgrounds}

We assume the spectral index $\alpha_{\mathrm{p}}=2,2.2,2.4$ and, as extreme case, $\alpha_{\mathrm{p}}=1.5$. As for the magnetic field $B \gg B_{\mathrm{CMB}}$ (see Eq. (7)), $B=1 \mu \mathrm{G}$, and $0.5 \mu \mathrm{G}$ (see Eq. (6)). The first choice of the magnetic field can be regarded as conservative considering that, for example, the volume-averaged magnetic field of Coma, the best-studied cluster for Faraday rotation measurements, is about $2 \mu \mathrm{G}$ (Bonafede et al. 2010); the latter should be considered optimistic with respect to current estimates. To clarify the meaning of the terms conservative/optimistic, note that the higher the magnetic field, the less room there is for protons, because the radio counts have to be respected, hence the lower the gamma-ray and neutrino fluxes.

For each $\alpha_{\mathrm{p}}$ and value of the magnetic field, the corresponding $P_{1}$ parameter is chosen in such a way that the computed $N_{1.4}\left(>F_{\text {min }}\right)$ does not overshoot the NVSS radio counts, and they are reported in Table 1. To make certain that our models respect current gamma-ray upper limits, the corresponding Coma-like and Perseus-like gamma-ray fluxes above $100 \mathrm{MeV}$ and $1 \mathrm{TeV}$, respectively, are also shown in Table 1, after assuming $M_{500}$ as in Reiprich \& Böhringer (2002), together with the total gamma-ray and neutrino flux at $100 \mathrm{MeV}$ and $250 \mathrm{TeV}$, respectively, for all the galaxy clusters in the Universe. All the reported values refer to $100 \%$ loud clusters, while the $30 \%$ case is studied only for $\alpha_{\mathrm{p}}=2$. (In the latter case, the remaining fraction of $70 \%$ quiet clusters are assumed to have an $L_{\gamma}(100 \mathrm{MeV})$ that is one order of magnitude lower than the loud ones.)

In the last column of Table 1 and for $\alpha_{\mathrm{p}} \geq 2$, we denote the cases that do not respect the gamma-ray upper limits on either Coma or Perseus by "G". For these cases, we recomputed $P_{1}$ so as to respect the Coma upper limit, our reference choice (see values in parenthesis in Table 1). However, our recomputed values for $\alpha_{\mathrm{p}}=2$ still overshoot the current Perseus gamma-ray upper limit. We nevertheless adopt the Coma upper limit as reference because it was calculated for $\alpha_{\mathrm{p}}=2$ and for a larger spatial extension, up to $R_{200}$. For $\alpha_{\mathrm{p}}=1.5$, the cases indicated by "N" in Table 1 exceed the IceCube neutrino data. Also in this case we recalculated $P_{1}$ to match the IceCube results after averaging over the corresponding energy range.

Figure 2 shows both the comparison of our models to the radio counts (on the left) and the computed gamma-ray (in black) and neutrino intensities (in red) as functions of the energy (on the right), for the chosen values of $\alpha_{\mathrm{p}}$ and $B$ assuming $100 \%$ loud clusters. For comparison, we plot the Fermi data (Fermi LAT Collaboration 2015a) and the IceCube $1 \sigma$ error band as in Aartsen et al. (2014a). The latter refers to the fouryear IceCube data sample. However, more recently a new fit has been provided, using two-year statistics but including low energy events down to $1 \mathrm{TeV}$. The best fit of the neutrino spectrum obtained in this case scales as $E_{v}^{-2.46}$ (Aartsen et al. 2015).

For $\alpha_{\mathrm{p}}>2$, both the gamma-ray and the neutrino diffuse backgrounds are well below the Fermi and the IceCube data in all cases. For $\alpha_{\mathrm{p}}=2$, while the gamma-ray flux is always lower than the Fermi measurements, the neutrino diffuse background could represent a significant fraction of the flux measured by IceCube for $B=1 \mu \mathrm{G}$ and $0.5 \mu \mathrm{G}$.

As known from radio observations, the case of $100 \%$ loud clusters is not realistic. Therefore, in Fig. 3, we show the same as in Fig. 2 for $\alpha_{\mathrm{p}}=2$, together with the more realistic case of $30 \%$ loud clusters. In the latter, galaxy clusters could make up at most about $10 \%(20 \%)$ of the neutrino flux measured by Ice Cube for $B=1 \mu \mathrm{G}(0.5 \mu \mathrm{G})$. This gives an estimation of how much our results for other spectral indices would change when moving from $100 \%$ loud clusters to the more realistic case of $30 \%$ loud clusters: $I_{\gamma, v, 30 \%} \approx I_{\gamma, v, 100 \%} / 2$ (see also Table 1 for comparison).

In the extreme case of $\alpha_{\mathrm{p}}=1.5$, we could explain the IceCube data by averaging over the corresponding energies for all cases, while respecting all other constraints from radio to gamma rays. However, we note that such a hard spectral index contradicts the most recent IceCube results, thus suggesting a softer spectral index (Aartsen et al. 2015).

Estimates of magnetic fields in clusters from Faraday rotation measurements range from $\sim \mu \mathrm{G}$ for merging clusters up to $10 \mu \mathrm{G}$ for cool-core clusters (Carilli \& Taylor 2002; Clarke 2004; Vogt \& Enßlin 2005; Bonafede et al. 2010, 2013). The case of $B=0.5 \mu \mathrm{G}$ should therefore be considered illustrative and optimistic because it contradicts current knowledge.

We conclude that, amongst all the cases we studied that respect both radio counts and current gamma-ray upper limits, hadronic interactions in galaxy clusters can realistically contribute at most up to $10 \%$ of the total extragalactic neutrino background, while contributing less than a few percentage points to the total extragalactic gamma-ray background. Moreover, the simplified requirement of not overshooting the NVSS radio counts on clusters leads to optimistic results. In fact, as explained in Sect. 2, not all the observed radio emission in clusters has a hadronic origin (Brunetti et al. 2012; Zandanel et al. 2014b). The open question is the exact contribution of protons to the nonthermal content of clusters, the corresponding contribution to the observed radio emission, and therefore, the possible gamma-ray emission (see Zandanel \& Ando 2014 for a discussion). This implies that even our results, which respect both NVSS counts and gamma-ray limits, should still be considered rather optimistic.

Finally, we note that, owing to our simplified approach using a gamma-ray luminosity-mass relation, the conclusions of this section can be generalised to any source of CR protons where these mix and hadronically interact with the ICM of galaxy clusters, such as those injected by structure formation shocks and AGNs. For any considered source of protons, the resulting secondary emission must respect both radio and gamma-ray constraints.

\subsection{Results: testing our standard assumptions}

To make our conclusions more robust, we comment in this section on two of our assumptions and on their effect on our final results: the redshift evolution and the value of the parameter $P_{2}$ in the luminosity-mass relation.

The redshift dependence has been omitted in Eq. (5). For the sake of completeness, we tested the effect of introducing a redshift dependence in the gamma-ray luminosity-mass relation by adopting $L_{\gamma} \propto \Omega_{\mathrm{m}}(1+z)^{3}+\Omega_{\Lambda}$, for $\alpha_{\mathrm{p}}=2.2,100 \%$ loud clusters and $B \gg B_{\mathrm{CMB}}$, roughly corresponding to the scaling observed in the Zandanel et al. (2014a) multi-frequency mockcluster catalogue for $L_{\gamma}(100 \mathrm{MeV})-M_{500}$. We found that omitting the redshift evolution causes both the radio counts and the high-energy fluxes to be only about $20 \%$ lower than the redshiftevolution case. Our results would scale accordingly, as would the $P_{1}$ parameter, and the maximum allowed contribution to the total extragalactic gamma-ray and neutrino fluxes would remain approximately the same. The case of radio counts is not as intuitive, but can be understood if noting that redshift evolution will 

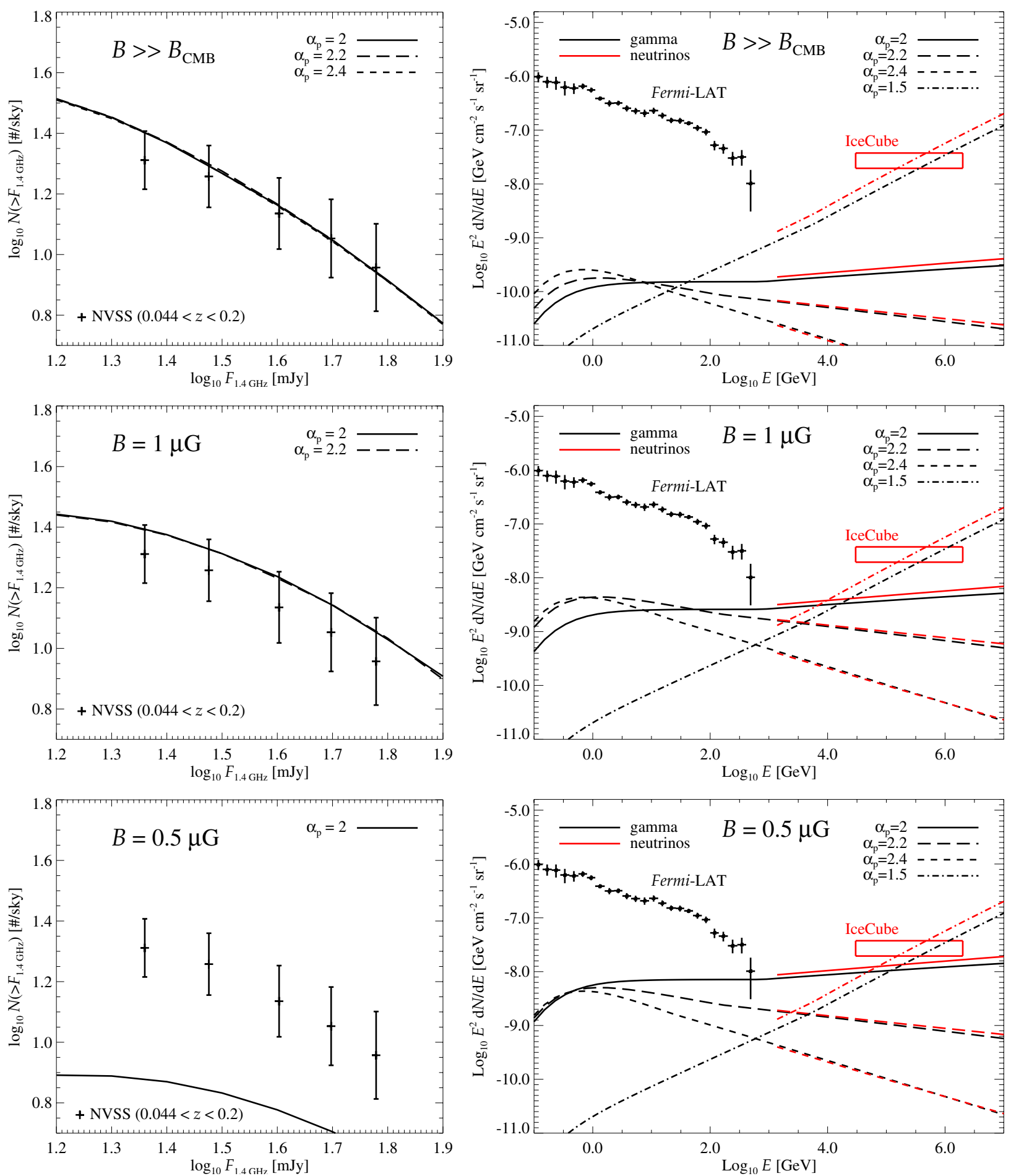

Fig. 2. Total gamma-ray and neutrino intensities (right) due to hadronic interactions in galaxy clusters, for $100 \%$ loud clusters, and the corresponding radio counts due to synchrotron emission from secondary electrons (left). From top to bottom, we plot the cases with $B \gg B_{\mathrm{CMB}}, B=1 \mu \mathrm{G}$ and $0.5 \mu \mathrm{G}$, respectively. For comparison, the Fermi (Fermi LAT Collaboration 2015a) and IceCube (Aartsen et al. 2014a) data are shown in the panels on the right. The neutrino intensity is meant for all flavours. All the plotted intensities respect NVSS radio counts and the gamma-ray upper limits on individual clusters. For $B=1 \mu \mathrm{G}$ and $\alpha_{\mathrm{p}}=2.4, B=0.5 \mu \mathrm{G}$ and $\alpha_{\mathrm{p}}=2.2,2.4$, and for $\alpha_{\mathrm{p}}=1.5$, the radio counts respecting the gamma-ray and neutrino limits, respectively, are below the $y$-scale range adopted for the panels on the left.

boost the luminosity of higher redshift objects, pushing them into a regime where they would be detectable and boosting the corresponding estimation of the radio counts, therefore requiring lower $P_{1}$ with respect to no-redshift evolution.

In Sect. 3.1, we fix the slope of the luminosity-mass relation to $P_{2}=5 / 3$, assuming that the hadronic-induced luminosity scales as the cluster thermal energy. In the conclusions of Sect. 3.2, we mentioned that our phenomenological approach can be generalised to any source of CR protons in clusters if these mix and hadronically interact with the ICM. However, while our standard choice for the $P_{2}$ parameter is appropriate for CR protons injected by structure formation shocks, it could be different for other CR sources. Clearly, a steeper slope would assign larger fluxes to high-mass objects that would easily 
F. Zandanel et al.: Gamma-ray and neutrino backgrounds from galaxy clusters
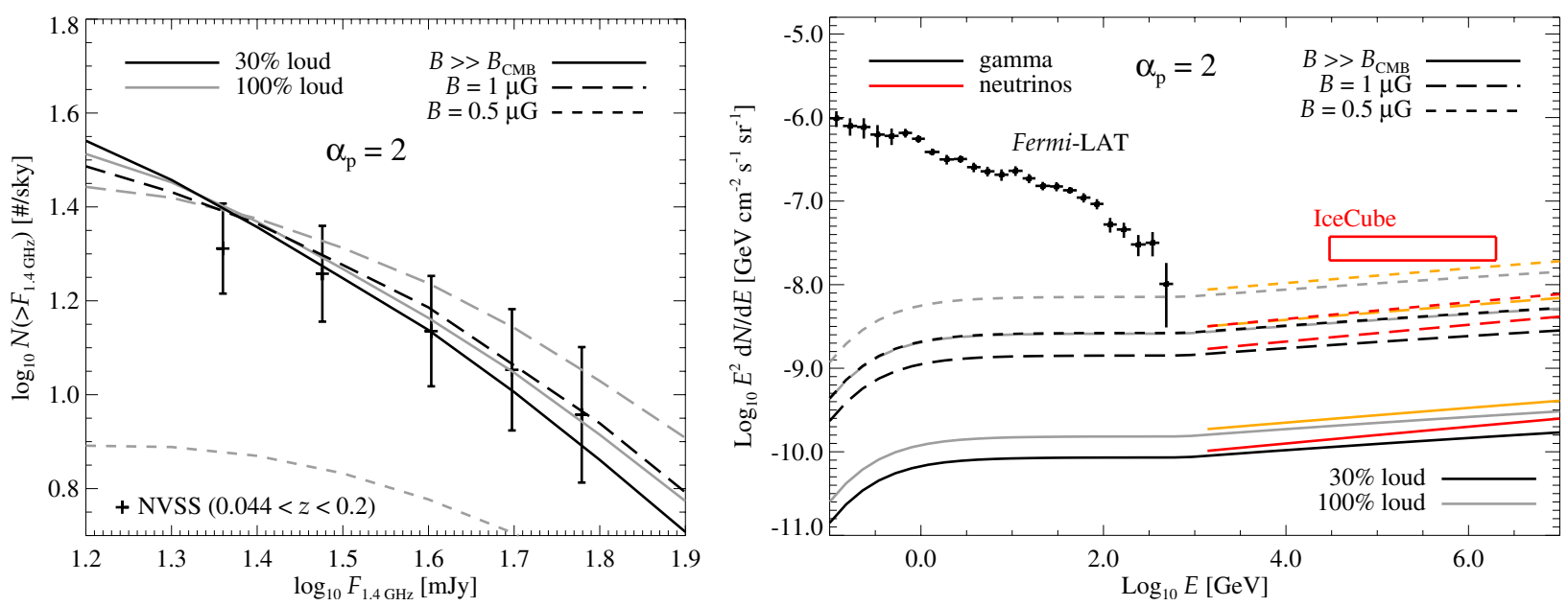

Fig. 3. Same as Fig. 3, together with the case of 30\% loud clusters for $\alpha_{\mathrm{p}}=2$. The remaining percentage of $70 \%$ quiet clusters has been assumed to have $L_{\gamma}(100 \mathrm{MeV})$ one order of magnitude lower than for the loud clusters. The $100 \%$ loud case is shown with lighter colours (i.e., in grey and orange).

overshoot radio counts. As a consequence, a lower value for $P_{1}$ would be allowed, and considering that low-mass clusters would have lower luminosities, we estimate that the total gamma-ray and neutrino fluxes would be lower than in the case of $P_{2}=5 / 3$, or at most, at the same level owing to the sum of a few very powerful, massive nearby sources.

To assess the changes obtained by assuming a flatter slope in the luminosity-mass relation, we tested the extreme value $P_{2}=1$ for $30 \%$ loud clusters, $\alpha_{\mathrm{p}}=2$ and $B=1 \mu \mathrm{G}$, our most optimistic, still realistic, case. We underline, however, that a luminositymass function with such a flat slope strongly contradicts current knowledge of the diffuse radio emission in galaxy clusters (Brunetti et al. 2009; Cassano et al. 2013). Either way, we found that, to respect radio counts, the maximum allowed contribution to the total extragalactic neutrino flux is about $15 \%$. This behaviour can be understood when noting again that such a flat slope implies that higher luminosities are assigned to lower mass clusters, pushing them into a regime where they would be detectable, hence boosting the corresponding radio counts. For the sake of completeness, we also added a redshift evolution of the luminosity as $(1+z)^{3}$ (as, e.g., for AGNs; Barger et al. 2005) to this extreme model that should eventually boost the neutrino production. We found that the maximum allowed contribution to the total extragalactic neutrino flux is $30 \%$ of the IceCube flux. We conclude that in all cases, the contribution to the total extragalactic gamma-ray flux is still negligible.

The estimation of a flux that is $30 \%$ of the IceCube one is the maximum that can be obtained under realistic conditions (30\% loud clusters, $B=1 \mu \mathrm{G}$ ) for the extreme value $P_{2}=1$ with $\alpha_{\mathrm{p}}=2$. The only way to additionally boost the total neutrino flux without changing the radio counts would be to integrate down to lower masses, as we also discuss in Sect. $4.3^{4}$. We note, however, that our standard lower mass bound is $M_{500, \lim }=$ $6.3 \times 10^{13} h^{-1} M_{\odot}=9 \times 10^{13} M_{\odot}$, roughly corresponding to $M_{200, \lim }=1.4 \times 10^{14} M_{\odot}$, and it already includes groups of galaxies. Extending the mass integration of the above case down

\footnotetext{
4 In Sect. 4.3 we also estimate the changes obtained by adopting the most recent Planck results for the cosmological parameters (Planck Collaboration XVI 2014). While for the semi-analytical model of the next section, the radio counts, total gamma-ray, and neutrino fluxes are enhanced by a factor of only about 1.7, in the phenomenological model with $P_{2}=1$, this would significantly boost the radio counts requiring the corresponding $P_{1}$ value to be lowered.
}

to $M_{500, \lim }=10^{13} h^{-1} M_{\odot}=1.4 \times 10^{13} M_{\odot}$, the $30 \%$ contribution to the total neutrino flux would become about $160 \%$, overshooting the IceCube measurement. One could, of course, finetune this mass limit to match the IceCube flux, but we think that such a combination of extreme parameters is highly unlikely. At any rate, the $E^{-2}$ spectrum is the only one for which such fine-tuning would give a significant total neutrino flux, and it disagrees with the latest IceCube results, suggesting a softer spectral index (Aartsen et al. 2015).

We conclude that the results of the phenomenological approach presented in Sect. 3.2 are robust against our assumptions and that they provide realistic estimates of the maximum allowed contribution of galaxy clusters to the total extragalactic gamma-ray and neutrino fluxes.

\section{Semi-analytical model for the cosmic-ray and intra-cluster-medium distributions}

In this section, we adopt a more sophisticated approach to modelling the CR and ICM distributions in galaxy clusters, as well as their magnetic field spatial distribution.

\subsection{Semi-analytical modelling}

For the ICM density distribution, we adopt the phenomenological model of Zandanel et al. (2014a), which is based on gas profiles obtained in X-rays (Croston et al. 2008) and on an observational correlation between gas fraction and mass of the clusters (Sun et al. 2009). This method allows a gas density to be assigned to any galaxy cluster using its mass alone, in such a way that the observed X-ray and Sunyaev-Zel'dovich scaling relations are correctly reproduced.

For the CR spatial and spectral distribution, we adopt the hadronic model proposed in Zandanel et al. (2014b), which extends the semi-analytical model of Pinzke \& Pfrommer (2010). The latter provides a scaling of the CR distribution with the cluster mass, while Zandanel et al. (2014b) introduced an effective parameterisation on the $\mathrm{CR}$ spatial distribution $\rho_{\mathrm{CR}}$ to account for CR transport phenomena. In all the models analysed in this section, we assume the proton spectral shape as in Pinzke \& Pfrommer (2010) where a universal CR spectrum is found amongst the simulated galaxy clusters. We rely 

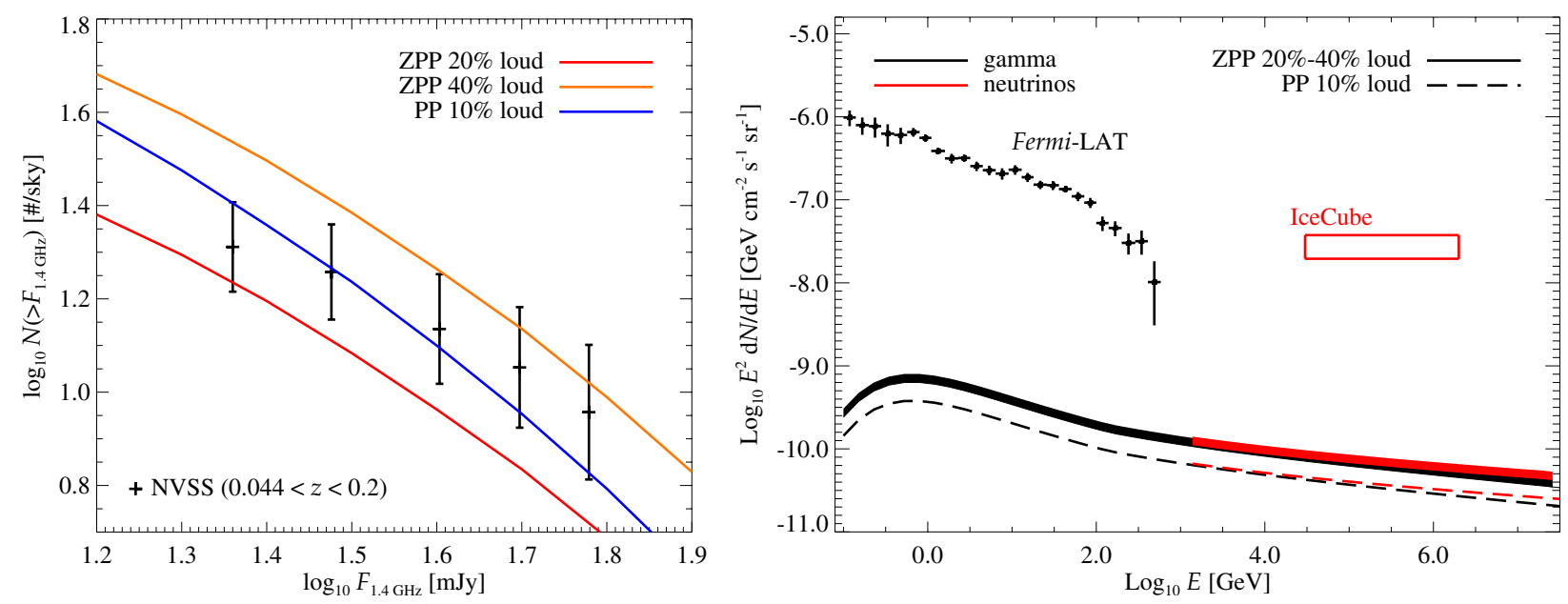

Fig. 4. Radio counts due to synchrotron emission of secondary electrons as from the semi-analytical model of Zandanel et al. (2014b, ZPP in the plots) on the left, and total gamma-ray and neutrino intensities on the right. For comparison, we plot the Fermi (Fermi LAT Collaboration 2015a) and IceCube data (Aartsen et al. 2014a) in the panels on the right. The neutrino intensity is meant for all flavours. We show the cases of the model applied to a mass function for $20 \%$ and $40 \%$ loud clusters, and additionally for $10 \%$ loud clusters with parameters as in the Pinzke \& Pfrommer (2010) model with a maximum CR proton acceleration efficiency of $15 \%$ (PP in the plots). According to this semi-analytical model, galaxy clusters contribute $<1 \%$ to the diffuse gamma-ray and neutrino backgrounds.

on Eqs. (4) and (4) with $L_{\gamma}\left(M_{500}, z\right)$ and $L_{1.4}\left(M_{500}, z\right)$ calculated by using Eqs. (2) and (1), with $\rho_{\mathrm{ICM}}$ and $\rho_{\mathrm{CR}}$ from the Zandanel et al. (2014a,b) models, including redshift evolution.

The cluster population is divided into $50 \%$ cool-core and $50 \%$ non-cool-core clusters (as from observations; see, e.g., Chen et al. 2007) with different parameterisation of the ICM and CR profiles. Cool-core clusters are relaxed objects, so CRs could stream out of the core, creating flat CR profiles. Non-coolcore clusters are more turbulent objects that should cause CRs to advect with the gas and create centrally peaked CR profiles. The difference between cool-core and non-cool-core clusters is modelled through the parameter $\gamma_{\mathrm{tu}}=\tau_{\mathrm{st}} / \tau_{\mathrm{tu}}$, i.e., the ratio between the characteristic time scale of streaming and that of turbulence. This parameter ranges from 100 for highly turbulent cluster and centrally peaked CR distributions to 1 for relaxed clusters and flat distributions as CRs move towards the outskirts (Zandanel et al. 2014b). Here, we assume $\gamma_{\mathrm{tu}}=3$ and 1 for loud and quiet cool-core clusters, and $\gamma_{\mathrm{tu}}=60$ and 1 for loud and quiet noncool-core clusters, respectively.

The magnetic field is assumed to radially scale as the gas density:

$B(r)=B_{0}\left(\frac{\rho_{\mathrm{ICM}}(r)}{\rho_{\mathrm{ICM}}(0)}\right)^{\alpha_{\mathrm{B}}}$,

where $B_{0}$ is the central magnetic field, and $\alpha_{\mathrm{B}}=0.5$ describes the declining rate of the magnetic field strength towards the cluster outskirts (Dubois \& Teyssier 2008; Bonafede et al. 2010; Kuchar \& Enßlin 2011, and references therein). In particular, for quiet clusters, we adopt a central magnetic field $B_{0}$ of $4 \mu \mathrm{G}$ (7.5 $\mu \mathrm{G}$ ) for non-cool-core (cool-core) clusters, while we choose $6 \mu \mathrm{G}(10 \mu \mathrm{G})$, to account for the potential turbulent dynamo in loud objects.

\subsection{Results: gamma-ray and neutrino backgrounds}

The model in Zandanel et al. (2014b, ZPP in tables and figures) reproduces the observed radio-to-X-ray and radio-to-SunyaevZel'dovich scaling relations of galaxy clusters and respects
Table 2. Total gamma-ray and neutrino fluxes for the semi-analytical model.

\begin{tabular}{lcc}
\hline \hline Model & $I_{\gamma}(100 \mathrm{MeV})$ & $I_{v}(250 \mathrm{TeV})$ \\
\hline ZPP 40\% & 3.0 & 1.3 \\
ZPP 20\% & 2.4 & 1.0 \\
ZPP 20\% z $2=0.6$ & 2.0 & 0.9 \\
ZPP 20\% M M00,lim $=10^{13} h^{-1} M_{\odot}$ & 6.2 & 2.3 \\
ZPP 20\% Planck & 4.2 & 1.7 \\
PP 10\% & 1.5 & 0.6 \\
\hline
\end{tabular}

Notes. Total gamma-ray and neutrino flux at $100 \mathrm{MeV}$ and $250 \mathrm{TeV}$ for the semi-analytical model in units of $10^{-8}$ and $10^{-21} \mathrm{~cm}^{-2} \mathrm{~s}^{-1} \mathrm{GeV}^{-1} \mathrm{sr}^{-1}$, respectively.

current gamma-ray upper limits ${ }^{5}$. In the left-hand panel of Fig. 4, we show the resulting radio counts for a fraction of $20 \%$ and $40 \%$ loud clusters. We find that the latter case should be considered extreme because hadronic interactions are known not to be uniquely responsible for the observed radio emission in clusters. Table 2 shows the corresponding total gamma-ray and neutrino fluxes.

Figure 4 (left panel) also shows the radio counts obtained by adopting $10 \%$ loud clusters with parameters corresponding to the model in Pinzke \& Pfrommer (2010, PP in tables and figures) with a maximum $\mathrm{CR}$ proton acceleration efficiency scaled down to $15 \%$ with respect to the originally assumed $50 \%$ in order to obey current gamma-ray constraints (Zandanel \& Ando 2014; Fermi LAT Collaboration 2014). For the remaining 90\% quiet fraction, the parameters of the previous model are assumed. The right-hand panel of Fig. 4 shows the corresponding total gammaray and neutrino intensities compared with the data from Fermi and IceCube. We conclude that galaxy clusters contribute less than $1 \%$ to the diffuse gamma-ray and neutrino backgrounds.

\footnotetext{
5 The parameters for the corresponding $L_{\gamma}(100 \mathrm{MeV})-M_{500}$ scaling relation at $z=0$ are $P_{1}=21.68$ and $P_{2}=1.62$ for non-coolcore clusters, and $P_{1}=22.41$ and $P_{2}=1.57$ for cool-core clusters. This translates in Coma-like and Perseus-like fluxes, for $\alpha_{\mathrm{p}}=2.2$, of $F(>100 \mathrm{MeV})=1.6 \times 10^{-9}$ and $F(>1 \mathrm{TeV})=7.6 \times 10^{-14} \mathrm{~cm}^{-2} \mathrm{~s}^{-1}$, respectively, below the current upper limits.
} 
F. Zandanel et al.: Gamma-ray and neutrino backgrounds from galaxy clusters
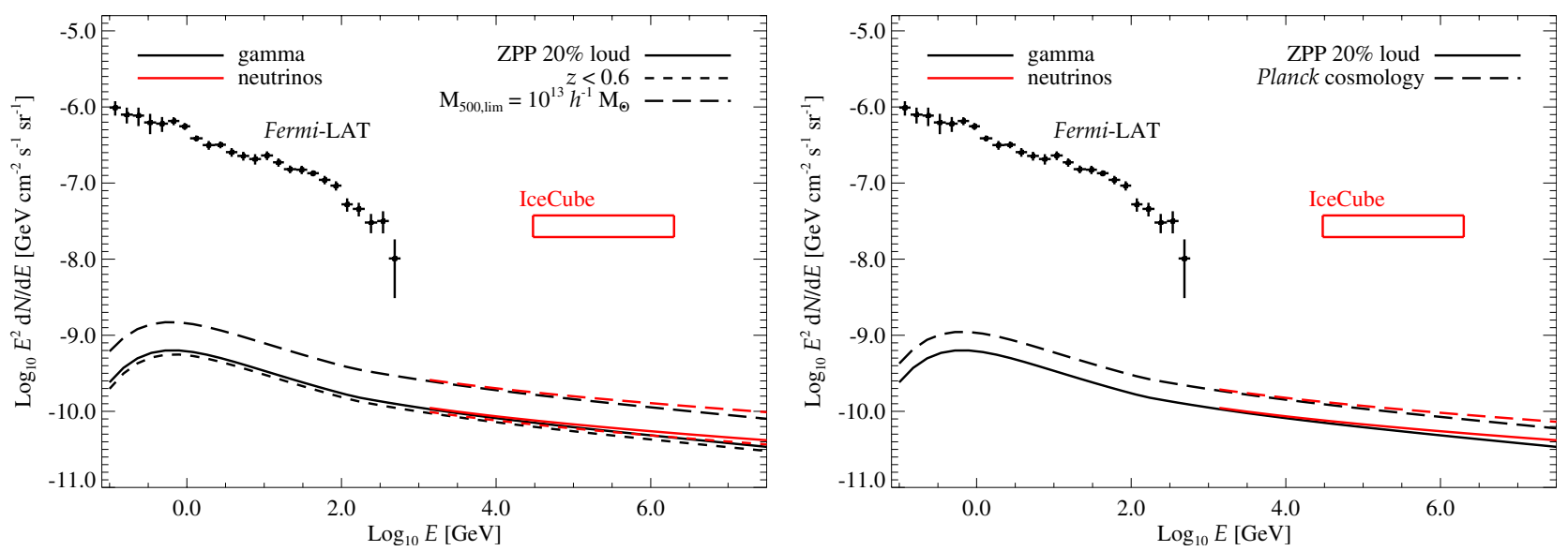

Fig. 5. Same as in the left panel of Fig. 4 for $20 \%$ loud clusters. The left panel shows the comparison with the previous model with one obtained adopting $z_{2}=0.6$, and one with a lower mass integration limit, of $10^{13} h^{-1} M_{\odot}$. The right panel shows the comparison with the model in Fig. 4 and one obtained using the Planck cosmological data.

The results reported in this section are more realistic than the ones shown in Sect. 3. However, we underline as the semianalytical model adopted here is based on the hypothesis that CRs are accelerated at structure formation shocks, while no assumption on the CR sources is made in the phenomenological approach of Sect. 3 .

\subsection{Results: dependence on cosmology and lower mass bound}

To test the robustness of our results, we computed the gammaray and neutrino backgrounds in the case of $20 \%$ loud clusters, first extending the integration down to lower masses $\left(M_{500, \lim }=\right.$ $\left.10^{13} h^{-1} M_{\odot}\right)$ and then adopting the most recent Planck results for the cosmological parameters.

The left-hand panel of Fig. 5 shows the gamma-ray and neutrino backgrounds for the same case as shown in Fig. 4 for $20 \%$ loud clusters with $M_{500, \lim }=10^{13.8} h^{-1} M_{\odot}$ and for $M_{500, \lim }=10^{13} h^{-1} M_{\odot}$. In the latter case, the gamma-ray and neutrino diffuse fluxes are significantly higher, while still representing less than $1 \%$ of the observational data. At the same time, the radio counts are exactly the same as in Fig. 4 since these are due to the higher mass objects. We additionally show the case with $M_{500, \text { lim }}=10^{13.8} h^{-1} M_{\odot}$ integrated up to $z_{2}=0.6$. As anticipated in Sect. 2, low-redshift objects represent the dominant contribution to the diffuse fluxes, because by adopting $z_{2}=0.6$, we obtain $82 \%$ of the total flux.

The right-hand panel of Fig. 5 shows gamma-ray and neutrino backgrounds for the same case as shown in Fig. 4 for $20 \%$ loud clusters and obtained by adopting the cosmological parameters determined by the Planck satellite (Planck Collaboration XVI 2014), i.e., $H_{0}=67.3 \mathrm{~km} \mathrm{~s}^{-1} \mathrm{Mpc}^{-1}, \Omega_{\mathrm{m}}=$ $0.32, \Omega_{\Lambda}=0.68$, and the corresponding mass function. The Planck cosmology results in an overall larger number of structures, as is clear in Fig. 1, therefore increasing both the total radio counts (not shown, but still below the $40 \%$ loud case of Fig. 4) and the total gamma-ray and neutrino fluxes. As shown in Fig. 5, the contribution to the extragalactic gamma-ray and neutrino background is at any rate lower than $1 \%$.

We note that the changes in $M_{500, \text { lim }}$ and in the cosmological parameters would affect the gamma-ray and neutrino diffuse fluxes obtained with the phenomenological approach in Sect. 3.2 approximately in the same way; i.e., they would increase by a factor of around 2, as can be seen from Table 2 .
Table 3. Maximum allowed neutrino flux from nearby clusters at $250 \mathrm{TeV}$.

\begin{tabular}{lcccc}
\hline \hline Cluster & & $\alpha_{\mathrm{p}}=2$ & $\alpha_{\mathrm{p}}=2.2$ & $\alpha_{\mathrm{p}}=2.4$ \\
\hline Virgo & $\leq$ & $3.2 \times 10^{-19}$ & $4.0 \times 10^{-20}$ & $3.4 \times 10^{-21}$ \\
Centaurus & $\leq$ & $7.3 \times 10^{-21}$ & $9.1 \times 10^{-22}$ & $7.7 \times 10^{-23}$ \\
Perseus & $\leq 1.8 \times 10^{-20}$ & $2.3 \times 10^{-21}$ & $1.9 \times 10^{-22}$ \\
Coma & $\leq 2.8 \times 10^{-20}$ & $3.5 \times 10^{-21}$ & $2.9 \times 10^{-22}$ \\
Ophiuchus & $\leq 4.5 \times 10^{-20}$ & $5.6 \times 10^{-21}$ & $4.7 \times 10^{-22}$ \\
\hline Sum & $\leq 4.2 \times 10^{-19}$ & $5.2 \times 10^{-20}$ & $4.4 \times 10^{-21}$ \\
\hline
\end{tabular}

Notes. Maximum allowed neutrino flux at $250 \mathrm{TeV}$ in units of $\mathrm{cm}^{-2} \mathrm{~s}^{-1} \mathrm{GeV}^{-1}$. Numbers were obtained assuming the phenomenological luminosity-mass relations of Sect. 3 . All cases refer to $B=1 \mu \mathrm{G}$; $\alpha_{\mathrm{p}}=2$ refers to $30 \%$ loud clusters, our most optimistic while still realistic case; and the cases of $\alpha_{\mathrm{p}}=2.2$ and 2.4 refer to $100 \%$ loud clusters (see Table 1).

\section{Comparison with stacking limits by IceCube and future detection prospects}

Recently, Aartsen et al. (2014b) have presented an all-sky pointand extended-source search with one-year IceCube data. In particular, they provide upper limits on a stacked sample of nearby galaxy clusters, namely Virgo, Centaurus, Perseus, Coma, and Ophiuchus, following predictions provided by Murase et al. (2008). Here, we focus on comparing with their "model B", where CR protons are supposed to be uniformly distributed within the cluster virial radius, and with their "isobaric model", where CRs are assumed to be distributed like the ICM in the clusters.

Following Abbasi et al. (2011), we estimate the summed output of the five aforementioned clusters to be $I_{v}(250 \mathrm{TeV})=$ $1.1 \times 10^{-20}$ and $1.6 \times 10^{-20} \mathrm{~cm}^{-2} \mathrm{~s}^{-1} \mathrm{GeV}^{-1}$ for Model $\mathrm{B}$ and the isobaric model, respectively. The latest results by Aartsen et al. (2014b) provide the following upper limits $I_{v, \mathrm{UL}}(250 \mathrm{TeV})=$ $6.9 \times 10^{-20}$ for Model B and $7.7 \times 10^{-20} \mathrm{~cm}^{-2} \mathrm{~s}^{-1} \mathrm{GeV}^{-1}$ for the isobaric model.

In Table 3, we provide the maximum allowed neutrino flux for the same five clusters by adopting the phenomenological luminosity-mass relations obtained in Sect. 3. We use the same 
mass of these clusters as from the literature ${ }^{6}$ in order to apply our $L_{\gamma}(100 \mathrm{MeV})-M_{500}$ relation, and they should therefore be considered indicative numbers, as in Sect. 3 for Coma and Perseus. We use $\alpha_{\mathrm{p}}=2,2.2$, and 2.4, omitting the extreme case of 1.5, and always refer to the case with $B=1 \mu \mathrm{G}$. For $\alpha_{\mathrm{p}}=2$, we adopt the $P_{1}$ value for $30 \%$ loud clusters. See Table 1 for more details.

The upper limits $I_{v, \mathrm{UL}}(250 \mathrm{TeV})$ for this stacked sample of clusters by Aartsen et al. (2014b) are obtained by assuming a spectral index of $\approx 2.15$, so we can compare with our results for $\alpha_{\mathrm{p}}=2.2$. From Table 3, we can see that the corresponding IceCube upper limits are just a factor of 1.3-1.5 above the maximum allowed flux for the stacked sample. When $\alpha_{\mathrm{p}}=2.4$, the maximum allowed flux for the stacked sample is one order of magnitude lower, while for $\alpha_{\mathrm{p}}=2$ it is one order of magnitude higher, with respect to $\alpha_{\mathrm{p}}=2.2$. We can conclude that, while special care should be used in considering the profile and extension of the possible signal, IceCube should be able to put constraints on our most optimistic case with $\alpha_{\mathrm{p}}=2$ and on the $\alpha_{\mathrm{p}}=2.2$ case in the very near future, while the case with $\alpha_{\mathrm{p}}=2.4$ is much harder to achieve.

We underline that the fluxes presented in this section for Virgo, Centaurus, Perseus, Coma, and Ophiuchus are quite optimistic for representing the maximum allowed by our phenomenological approach. For example, we know that the fluxes of Virgo, Centaurus, and Ophiuchus should lie significantly below the loud part of the luminosity-mass relation owing to the lack of diffuse radio emission in Virgo and Centaurus, and to the very low surface-brightness radio emission observed in Ophiuchus (see, e.g., Zandanel et al. 2014b), pushing also the possible hadronic-induced gamma-ray and neutrino fluxes to lower levels. Any realistic modelling of these objects should consider this evidence carefully. In fact, the stacked signal from the five nearby clusters presented in this section already significantly overshoots the total signal obtained with the more realistic modelling of the CR proton population in clusters performed in Sect. 4 with our semi-analytical approach.

\section{Proton-photon interactions in galaxy clusters}

Besides interacting with the ICM, relativistic protons in clusters of galaxies can also interact with the ambient photon fields. The two main interaction processes are electron-positron pair production $\left(\mathrm{p}+\gamma \rightarrow \mathrm{p}+\mathrm{e}^{+}+\mathrm{e}^{-}\right)$and photomeson production. (Close to the threshold, the dominant contribution comes from the resonant channel: $\mathrm{p}+\gamma \rightarrow \Delta^{+} \rightarrow \mathrm{p}+\pi^{0}$ or $n+\pi^{-}$.) Both photons and neutrinos are expected in photomeson production owing to the decay of neutral and charged pions, respectively (Kelner \& Aharonian 2008). Thus, this is another channel to be investigated for assessing the contribution of clusters of galaxies to the diffuse neutrino flux observed by IceCube.

The process of photomeson production has a kinematic threshold and takes place when the energy of the photon in the rest frame of the proton exceeds $E_{\mathrm{thr}} \simeq 145 \mathrm{MeV}$ (see, e.g., Kelner \& Aharonian 2008). The most prominent radiation field in clusters of galaxies is the CMB (e.g., Pinzke et al. 2011), whose photons have a typical energy of $E_{\mathrm{CMB}} \approx 7 \times 10^{-4} \mathrm{eV}$. The threshold energy for a proton to produce a meson is $E_{\mathrm{p}, \mathrm{thr}}=$ $E_{\mathrm{thr}}^{2} / 2 E_{\mathrm{CMB}} \approx 10^{20} \mathrm{eV}$, but in fact protons with slightly smaller energy can also interact with the high-energy tail of the black

\footnotetext{
6 The mass $M_{500}$ for Centaurus, Perseus, Coma, and Ophiuchus is taken from Reiprich \& Böhringer (2002), while for Virgo it is derived from Pinzke et al. (2011).
}

body radiation (Greisen 1966; Zatsepin \& Kuz'min 1966). Thus, one can conclude that proton-photon interactions in clusters of galaxies can contribute to the high-energy neutrino background only if protons with energy in excess of several $10^{19} \mathrm{eV}$ are present in the ICM.

Accretion shocks around clusters of galaxies have been proposed as the sites of the acceleration of ultrahigh-energy CRs, the main reason being that their very large size (Mpc scale) would allow the acceleration and confinement of protons of ultrahigh energies (e.g., Norman et al. 1995). An estimate of the maximum energy achievable by protons at cluster accretion shocks can be obtained by equating the acceleration time, computed in the framework of diffusive shock acceleration, to the energy loss time due to proton-photon interactions. Accurate calculations have shown that the maximum energy of protons is determined by the energy losses due to electron-positron pair production and that for the most optimistic assumptions it ranges from a few $10^{18} \mathrm{eV}$ to a few $10^{19} \mathrm{eV}$ (Vannoni et al. 2011). Because they are cooled mainly by pair production, protons are thus not expected to produce any appreciable flux of neutrinos through the proton-photon interaction channel. Heavy nuclei, such as iron, can be accelerated up to $\approx 10^{20} \mathrm{eV}$ at cluster accretion shocks (e.g., Allard \& Protheroe 2009; Vannoni et al. 2011). However, iron cools mainly by photodisintegration in a soft photon field, and in this case the neutrino yield is very suppressed compared to the case of photomeson production (Kotera et al. 2009).

Another possible scenario for the production of neutrinos in the ICM would be to assume that clusters contain sources of ultrahigh-energy CRs. This would lead to two advantages. First of all, the infrared photon background in the cluster core would be enhanced with respect to the cosmological one thanks to the contribution from the galaxies in the cluster (Lagache et al. 2005; Pinzke et al. 2011). Second, the turbulent magnetic field present within the ICM would partially confine ultrahigh-energy protons, enhancing the probability of interaction. These two facts would increase the expected neutrino flux from proton-photon interactions (e.g., de Marco et al. 2006; Kotera et al. 2009). However, the source of ultrahigh-energy CRs will have to be located in the centre of the cluster, where the infrared photon background is enhanced and the confinement of protons is more effective (thanks to a larger magnetic field). As pointed out in Kotera et al. (2009), the high gas density in the core of clusters would also enhance the probability of proton-proton interactions, which would dominate the neutrino production below energies of $\approx 10^{18} \mathrm{eV}$.

Finally, it has to be noticed that the expected spectrum of neutrinos from photopion production interactions is significantly harder than $E^{-2}$ below the energy threshold, which is at odds with the evidence for a spectral index softer than two revealed by IceCube (Murase et al. 2013; Becker Tjus et al. 2014). This implies that proton-photon interactions make a negligible contribution to the neutrino flux in the energy domain of the IceCube neutrinos.

\section{Contribution to the small-scale anisotropies of the gamma-ray background}

Recently, Fermi LAT Collaboration (2012) has analysed the anisotropies in the EGB and found an excess in its angular power spectrum over what is expected with a completely diffuse source distribution on multipole ranges $155 \leq \ell \leq 504$ (corresponding to $\lesssim 2^{\circ}$ angular scales). For the first time, this 
has shown that a major fraction of the EGB is made by discrete sources, and, in fact, Cuoco et al. (2012) point out that the measured level of anisotropies is consistent with predictions for gamma-ray blazars (Ando et al. 2007). They also obtained the upper limit on the angular power spectrum as $C_{\ell}<3.3 \times$ $10^{-18}\left(\mathrm{~cm}^{-2} \mathrm{~s}^{-1} \mathrm{sr}^{-1}\right)^{2}$ sr for $155 \leq \ell \leq 504$ and $E=1-10 \mathrm{GeV}$ on other source components, after subtracting the main blazar contribution. Even though clusters are not the dominant contributors to the isotropic component of the diffuse gamma-ray and neutrino backgrounds (as shown in the previous sections), they may make substantial contributions to the EGB anisotropies. In particular, since there are relatively fewer than other astrophysical sources, such as star-forming galaxies, the cluster component in the EGB should be more anisotropic. To this end, we estimate the cluster contribution to the EGB anisotropies in this section and compare it to the Fermi data at sub-degree angular scales.

The angular power spectrum coming from proton-proton interactions in galaxy clusters can be calculated as follows (e.g., Ando et al. 2007):

$C_{\ell}=\int \frac{\mathrm{d} \chi}{\chi^{2}} W_{\gamma}^{2}(E[1+z], z) P_{\mathrm{C}}\left(k=\frac{\ell}{\chi}, \chi\right)$,

where $\chi$ is the comoving distance (we use the same redshift range as in previous sections), $W_{\gamma}=(1+z)^{3} A_{\gamma}(E[1+z]) / 4 \pi$ is the so-called window function, and $P_{\mathrm{C}}(k, \chi)$ is the power spectrum for the cluster gamma-ray emission. The last can be divided into one- and two-halo terms, $P_{\mathrm{C}}=P_{\mathrm{C}}^{1 \mathrm{~h}}+P_{\mathrm{C}}^{2 \mathrm{~h}}$, which we express as (e.g., Komatsu \& Seljak 2002; Ando et al. 2007)

$$
\begin{aligned}
P_{\mathrm{C}}^{1 \mathrm{~h}}= & \int \mathrm{d} M \frac{\mathrm{d} n}{\mathrm{~d} M}\left[\int 4 \pi r^{2} \mathrm{~d} r \rho_{\mathrm{CR}}(r) \rho_{\mathrm{gas}}(r) \frac{\sin (k r)}{k r}\right]^{2} \\
P_{\mathrm{C}}^{2 \mathrm{~h}}= & {\left[\int \mathrm{d} M \frac{\mathrm{d} n}{\mathrm{~d} M} b(M, z) \int 4 \pi r^{2} \mathrm{~d} r \rho_{\mathrm{CR}}(r) \rho_{\mathrm{gas}}(r) \frac{\sin (k r)}{k r}\right]^{2} } \\
& \times P_{\mathrm{lin}}(k, \chi)
\end{aligned}
$$

respectively, where the radial integration goes up to $R_{500}$. In the two-halo term, we assume that the linear matter power spectrum $P_{\text {lin }}(k, \chi)$ is related to the cluster power spectrum via the linear bias $b(M, z)$ (Tinker et al. 2010). We find that the one-halo term dominates the two-halo term at all multipoles $\ell$.

In Fig. 6, we show the angular power spectrum for the semianalytical models of Sect. 4 for $20 \%$ and $40 \%$ loud clusters integrated in the energy bin from 1 to $10 \mathrm{GeV}$. We compare with the measurement on the EGB by Fermi (Fermi LAT Collaboration 2012) and upper limits by Cuoco et al. (2012). We compare $C_{\ell}^{1 / 2}$ instead of $C_{\ell}$. This is because $C_{\ell}$ is a variance, so if each cluster is twice as bright, then $C_{\ell}$ becomes larger by a factor of 4. Therefore, taking the square-root will reflect the correct scaling with respect to the cluster contribution. Our prediction is about one order of magnitude less than the Fermi upper limit. This means that in scenarios where the total galaxy cluster intensity is much higher than in the models of Sect. 4, as is potentially realised for some of the simple phenomenological models discussed in Sect. 3, the angular power spectrum could be a powerful discriminator, as powerful as radio counts. Additionally, there are other contributions to the EGB anisotropies that would further increase the gamma-ray angular power spectrum, such as, but not only, dark matter annihilation (e.g., Ando \& Komatsu 2006, 2013; Fornasa et al. 2013; Fermi LAT Collaboration 2015b), further exacerbating the possible tension with the upper limits by Cuoco et al. (2012).

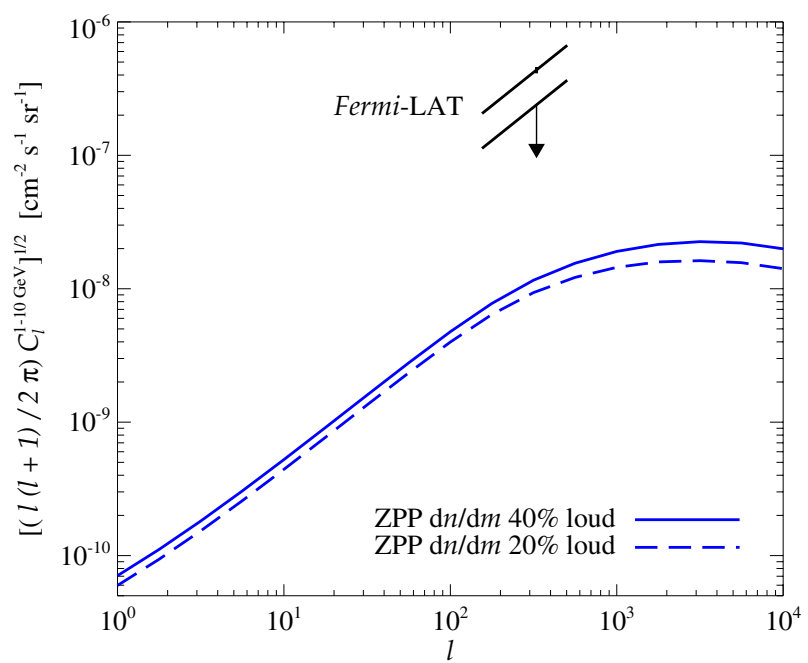

Fig. 6. Gamma-ray angular power spectrum for emission resulting from proton-proton interactions in galaxy clusters in the energy range $1-10 \mathrm{GeV}$. We show the result for the semi-analytical model of Sect. 4 for $20 \%$ and $40 \%$ loud clusters. We plot the EGB anisotropy measured by Fermi (Fermi LAT Collaboration 2012) for comparison, which is explained by unresolved blazars, and the upper limits obtained once the blazar component is subtracted (Cuoco et al. 2012). We plot the square root of $\ell(\ell+1) C_{\ell} / 2 \pi$, which implies that the shown quantity is directly proportional to an increase in intensity.

\section{Discussion and conclusions}

In this work we estimated the contribution from hadronic protonproton interactions in galaxy clusters to the total extragalactic gamma-ray and neutrino fluxes, while including radio constraints for the first time. We modelled the cluster population by means of their mass function. Our approach makes use of a phenomenological luminosity-mass relation applied to all clusters, constructed by requiring radio counts to be respected. We adopted four different proton spectral indices $\alpha_{\mathrm{p}}=1.5,2,2.2$, and 2.4, and three different magnetic field values $B \gg B_{\mathrm{CMB}}$, $B=1 \mu \mathrm{G}$, and $B=0.5 \mu \mathrm{G}$. The last is meant to only be an illustrative case, because it contrasts with current estimates of magnetic fields in clusters.

Radio observations reveal that not all galaxy clusters host diffuse synchrotron radio emission, with upper limits about an order of magnitude below the loud state (Brunetti et al. 2009; Cassano et al. 2013). For the sake of simplicity, we adopted $100 \%$ loud clusters, leading to an optimistic estimation. However, we also discussed the case with $30 \%$ loud clusters for $\alpha_{\mathrm{p}}=2$, corresponding to our most optimistic case, according to recent estimates of the loud fraction. In our phenomenological model, the slope of the luminosity-mass relation is fixed to $5 / 3$, assuming that the hadronic-induced luminosity scales as the cluster thermal energy, and the redshift evolution was omitted for simplicity. We showed that our assumptions are robust, and we estimated that ignoring the redshift evolution results in only about a $20 \%$ underestimation of the radio counts and total high-energy fluxes.

By requiring all the current constraints to be respected from radio counts to gamma-ray upper limits on individual clusters, we showed that galaxy clusters can contribute at most up to $10 \%$ of the total neutrino background for $\alpha_{\mathrm{p}}=2$, while contributing much less to the EGB. For $\alpha_{\mathrm{p}}>2$, the gamma-ray and neutrino backgrounds in all considered cases are $<1 \%$ of the gamma-ray and neutrino fluxes measured by Fermi (Fermi LAT Collaboration 2010b, 2015a) and IceCube 
(Aartsen et al. 2014a), respectively. Only for the extreme case with $\alpha_{\mathrm{p}}=1.5$ is the neutrino flux of the same order of magnitude as the IceCube data; however, such a hard spectral shape contrasts with the most recent IceCube spectral fit of neutrino flux (Aartsen et al. 2015).

We also adopted a more refined approach that employs a semi-analytical model where the ICM density is constructed from X-ray observations, and the CR spatial and spectral distribution is based on state-of-art hydrodynamic simulations (Zandanel et al. 2014b). In this case, we divided the cluster population into cool-core/non-cool-core and loud/quiet subsamples, as suggested by observations, where the transition from the loud to the quiet state is achieved through a change in the $\mathrm{CR}$ propagation properties. We find that galaxy clusters contribute to $<1 \%$ to the EGB and to the neutrino flux measured by IceCube. While this semi-analytical model is more realistic than the simplified phenomenological model discussed above, we assume in this case that CRs are accelerated at structure formation shocks, while no assumption on the CR sources was made in the phenomenological approach.

We then compared the flux of five nearby clusters - Virgo, Centaurus, Perseus, Coma and Ophiuchus - to recent results by IceCube (Aartsen et al. 2014b). The IceCube upper limits are just a factor 1.5 above our maximum allowed (stacked) flux for these objects for the case of $\alpha_{\mathrm{p}}=2.2$, which compares well to what is used in Aartsen et al. (2014b). We showed that, despite the small contribution to the total neutrino flux, IceCube should be able to put constraints on our most optimistic case with $\alpha_{\mathrm{p}}=2$, and very soon in the case with $\alpha_{\mathrm{p}}=2.2$, using the stacked sample of nearby massive clusters.

We briefly also discussed the case of proton-photon interactions in galaxy cluster. We found that this channel gives a negligible contribution to the expected neutrino flux in the multi TeVPeV energy domain.

While galaxy clusters represent a sub-dominant contribution to the EGB, they could substantially contribute to its anisotropy because they are fewer in number than other astrophysical sources and, therefore, are expected to be more anisotropic. For this reason, we computed the angular power spectrum for the considered semi-analytical models and showed that the amplitude of the angular fluctuations, represented by $C_{\ell}^{1 / 2}$, is about one order of magnitude below the Fermi upper limits.

We conclude that there is no realistic scenario in which galaxy clusters can contribute substantially to either the EGB or the extragalactic neutrino flux, since the maximum contribution is at most $10 \%$ in the simple phenomenological modelling, while it is less than $1 \%$ in most cases and in the more realistic semianalytical modelling. We also proved that our conclusions are not significantly affected by our assumptions. Our results therefore put earlier works into prospective, which turned out to be overly optimistic in estimating the galaxy cluster contribution (e.g., Loeb \& Waxman 2000; Murase et al. 2013).

We would like to conclude with a few additional comments on our assumptions. In our calculations, we omitted both a possible cut-off in the CR spectrum at high energies caused by protons that are no longer confined to the cluster and the absorption of high-energy gamma rays due to interactions with the extragalactic background light. The former implies larger high-energy neutrino fluxes, while the latter implies slightly optimistic gammaray fluxes. Additionally, we stress once more how requiring the synchrotron emission from secondary electrons not to overshoot radio counts also results in rather optimistic gamma-ray and neutrino fluxes. This is because so-called giant radio haloes hosted in merging, non-cool-core clusters cannot be explained solely by hadronic emission (Brunetti et al. 2012; Zandanel et al. 2014b). Therefore, the secondary emission seems to represent only a fraction of the total observed radio emission.

As a final note on the semi-analytical modelling, we underline that the transition from the loud to the quiet state in the galaxy cluster population is not achieved in the classical hadronic model, meaning that it predicts that all clusters should have the same level of secondary emission. This clearly contradicts observations and represents one of the problems with the hadronic scenario (see Enßlin et al. 2011 for a discussion). The only mechanism that has been proposed so far to solve this problem is to vary CR propagation properties (see, e.g., Wiener et al. 2013), which was also adopted in our semi-analytical approach through the Zandanel et al. (2014b) model. We note, however, that it is still being debated whether the conditions for CR diffusion can be reached in the ICM. In the worst-case scenario, the secondary electrons produced in proton-proton collisions in clusters would only be seed electrons for subsequent turbulent re-acceleration (see, e.g., Brunetti \& Lazarian 2011; Brunetti et al. 2012). This would imply a much lower secondary emission only at the level of the quiet state. If this turns out to be the case, the total gamma-ray and neutrino fluxes from galaxy clusters should be even lower than what we have estimated here.

Acknowledgements. We thank the anonymous referee for useful comments. We thank Denis Allard, Rossella Cassano, and Kohta Murase for useful discussions. This work was supported by the Netherlands Organisation for Scientific Research (NWO) through a Vidi grant (S.A., I.T., and F.Z.) and a PHC Van Gogh grant (S.G.)

\section{References}

Aartsen, M. G., Abbasi, R., Abdou, Y., et al. 2013, Phys. Rev. Lett., 111, 021103 Aartsen, M. G., Ackermann, M., Adams, J., et al. 2014a, Phys. Rev. Lett., 113, 101101

Aartsen, M. G., Ackermann, M., Adams, J., et al. 2014b, ApJ, 796, 109

Aartsen, M. G., Ackermann, M., Adams, J., et al. 2015, Phys. Rev. D, 91, 022001

Abbasi, R., Abdou, Y., Abu-Zayyad, T., et al. 2011, ApJ, 732, 18

Abdo, A. A., Ackermann, M., Ajello, M., et al. 2010, ApJ, 720, 435

Ackermann, M., Ajello, M., Allafort, A., et al. 2012, ApJ, 755, 164

Ahlers, M., \& Murase, K. 2014, Phys. Rev. D, 90, 023010

Ajello, M., Gasparrini, D., Sanchez-Conde, M., et al. 2015, ApJ, 800, L27

Allard, D., \& Protheroe, R. J. 2009, A\&A, 502, 803

Anchordoqui, L. A., Goldberg, H., Halzen, F., \& Weiler, T. J. 2004, Phys. Lett., 600, 202

Anchordoqui, L. A., Barger, V., Cholis, I., et al. 2014a, J. High Energy Astrophys., 1, 1

Anchordoqui, L. A., Goldberg, H., Paul, T. C., da Silva, L. H. M., \& Vlcek, B. J 2014b, Phys. Rev. D, 90, 123010

Anchordoqui, L. A., Paul, T. C., da Silva, L. H. M., Torres, D. F., \& Vlcek, B. J. 2014c, Phys. Rev. D, 89, 127304

Ando, S., \& Komatsu, E. 2006, Phys. Rev. D, 73, 023521

Ando, S., \& Komatsu, E. 2013, Phys. Rev. D, 87, 123539

Ando, S., \& Nagai, D. 2008, MNRAS, 385, 2243

Ando, S., \& Nagai, D. 2012, J. Cosmol. Astro-Part. Phys., 7, 17

Ando, S., Komatsu, E., Narumoto, T., \& Totani, T. 2007, Phys. Rev. D, 75, 063519

Barger, A. J., Cowie, L. L., Mushotzky, R. F., et al. 2005, ApJ, 129, 578

Becker Tjus, J., Eichmann, B., Halzen, F., Kheirandish, A., \& Saba, S. M. 2014 Phys. Rev. D, 89, 123005

Berezinsky, V. S., Blasi, P., \& Ptuskin, V. S. 1997, ApJ, 487, 529

Blasi, P., \& Colafrancesco, S. 1999, Astropart. Phys., 12, 169

Blasi, P., Gabici, S., \& Brunetti, G. 2007, Int. J. Mod. Phys. A, 22, 681

Bonafede, A., Feretti, L., Murgia, M., et al. 2010, A\&A, 513, A30

Bonafede, A., Vazza, F., Brüggen, M., et al. 2013, MNRAS, 433, 3208

Brunetti, G., \& Jones, T. W. 2014, Int. J. Mod. Phys. D, 23, 30007

Brunetti, G., \& Lazarian, A. 2011, MNRAS, 410, 127

Brunetti, G., Cassano, R., Dolag, K., \& Setti, G. 2009, A\&A, 507, 661

Brunetti, G., Blasi, P., Reimer, O., et al. 2012, MNRAS, 426, 956

Carilli, C. L., \& Taylor, G. B. 2002, ARA\&A, 40, 319

Cassano, R., Brunetti, G., Röttgering, H. J. A., \& Brüggen, M. 2010, A\&A, 509, A68 
Cassano, R., Brunetti, G., Norris, R. P., et al. 2012, A\&A, 548, A100

Cassano, R., Ettori, S., Brunetti, G., et al. 2013, ApJ, 777, 141

Chakraborty, S., \& Izaguirre, I. 2015, Phys. Lett. B, 745, 35

Chang, X.-C., \& Wang, X.-Y. 2014, ApJ, 793, 131

Chen, Y., Reiprich, T. H., Böhringer, H., Ikebe, Y., \& Zhang, Y.-Y. 2007, A\&A, 466, 805

Clarke, T. E. 2004, J. Kor. Astron. Soc., 37, 337

Croston, J. H., Pratt, G. W., Böhringer, H., et al. 2008, A\&A, 487, 431

Cuoco, A., Komatsu, E., \& Siegal-Gaskins, J. M. 2012, Phys. Rev. D, 86, 063004

de Marco, D., Hansen, P., Stanev, T., \& Blasi, P. 2006, Phys. Rev. D, 73, 043004 Dennison, B. 1980, ApJ, 239, L93

Dermer, C. D. 2007, AIP Conf. Proc., 921, 122

Di Mauro, M., \& Donato, F. 2015, Phys. Rev. D, submitted [arXiv: 1501.05316]

Di Mauro, M., Calore, F., Donato, F., Ajello, M., \& Latronico, L. 2014a, ApJ, 780,161

Di Mauro, M., Donato, F., Lamanna, G., Sanchez, D. A., \& Serpico, P. D. 2014b, ApJ, 786, 129

Domínguez, A., Primack, J. R., Rosario, D. J., et al. 2011, MNRAS, 410, 2556

Dubois, Y., \& Teyssier, R. 2008, A\&A, 482, L13

Enßlin, T., Pfrommer, C., Miniati, F., \& Subramanian, K. 2011, A\&A, 527, A99

Esmaili, A., \& Serpico, P. D. 2013, J. Cosmol. Astro-Part. Phys., 1311, 054

Esmaili, A., Kang, S. K., \& Serpico, P. D. 2014, J. Cosmol. Astro-Part. Phys., 12,054

Feldstein, B., Kusenko, A., Matsumoto, S., \& Yanagida, T. T. 2013, Phys. Rev. D, 88, 015004

Feretti, L., Giovannini, G., Govoni, F., \& Murgia, M. 2012, A\&ARv, 20, 54

Fermi LAT Collaboration 2010a, ApJ, 717, L71

Fermi LAT Collaboration 2010b, Phys. Rev. Lett., 104, 101101

Fermi LAT Collaboration 2012, Phys. Rev. D, 85, 083007

Fermi LAT Collaboration 2014, ApJ, 787, 18

Fermi LAT Collaboration 2015a, ApJ, 799, A86

Fermi LAT Collaboration 2015b, J. Cosmol. Astro-Part. Phys., submitted, [arXiv: 1501.05464]

Fichtel, C. E., Hartman, R. C., Kniffen, D. A., et al. 1977, ApJ, 217, L9

Fornasa, M., Zavala, J., Sánchez-Conde, M. A., et al. 2013, MNRAS, 429, 1529

Fox, D., Kashiyama, K., \& Meszaros, P. 2013, ApJ, 774, 74

Gabici, S., \& Blasi, P. 2003, Astropart. Phys., 19, 679

Giovannini, G., Tordi, M., \& Feretti, L. 1999, New Astron., 4, 141

Greisen, K. 1966, Phys. Rev. Lett., 16, 748

Griffin, R. D., Dai, X., \& Kochanek, C. S. 2014, ApJ, 795, L21

Halzen, F., \& Hooper, D. 2005, Astropart. Phys., 23, 537

He, H.-N., Wang, T., Fan, Y.-Z., Liu, S.-M., \& Wei, D.-M. 2013, Phys. Rev. D, 87, 063011

H.E.S.S. Collaboration. 2009a, A\&A, 502, 437

H.E.S.S. Collaboration. 2009b, A\&A, 495, 27

Huber, B., Tchernin, C., Eckert, D., et al. 2013, A\&A, 560, A64

Hümmer, S., Baerwald, P., \& Winter, W. 2012, Phys. Rev. Lett., 108, 231101

Joshi, J. C., Winter, W., \& Gupta, N. 2014, MNRAS, 439, 3414

Kale, R., Venturi, T., Giacintucci, S., et al. 2013, A\&A, 557, A99

Kashiyama, K., \& Meszaros, P. 2014, ApJ, 790, L14

Katz, B., Waxman, E., Thompson, T., \& Loeb, A. 2013, arXiv e-prints [arXiv: 1311.0287]

Kelner, S. R., \& Aharonian, F. A. 2008, Phys. Rev. D, 78, 034013

Kelner, S. R., Aharonian, F. A., \& Bugayov, V. V. 2006, Phys. Rev. D, 74, 034018

Keshet, U., Waxman, E., Loeb, A., Springel, V., \& Hernquist, L. 2003, ApJ, 585, 128

Komatsu, E., \& Seljak, U. 2002, MNRAS, 336, 1256

Komatsu, E., Smith, K. M., Dunkley, J., et al. 2011, ApJS, 192, 18

Kotera, K., Allard, D., Murase, K., et al. 2009, ApJ, 707, 370

Kuchar, P., \& Enßlin, T. A. 2011, A\&A, 529, A13

Kushnir, D., \& Waxman, E. 2009, J. Cosmol. Astro-Part. Phys., 8, 2

Lacki, B. C., Thompson, T. A., Quataert, E., Loeb, A., \& Waxman, E. 2011, ApJ, 734, 107
Lagache, G., Puget, J.-L., \& Dole, H. 2005, ARA\&A, 43, 727

Liu, R.-Y., \& Wang, X.-Y. 2013, ApJ, 766, 73

Liu, R.-Y., Wang, X.-Y., Inoue, S., Crocker, R., \& Aharonian, F. 2014, Phys. Rev. D, 89, 083004

Loeb, A., \& Waxman, E. 2000, Nature, 405, 156

Loeb, A., \& Waxman, E. 2006, J. Cosmol. Astro-Part. Phys., 0605, 003

MAGIC Collaboration. 2010, ApJ, 710, 634

MAGIC Collaboration. 2012, A\&A, 541, A99

Miniati, F., Ryu, D., Kang, H., \& Jones, T. W. 2001, ApJ, 559, 59

Murase, K., \& Beacom, J. F. 2013, J. Cosmol. Astro-Part. Phys., 2, 28

Murase, K., \& Ioka, K. 2013, Phys. Rev. Lett., 111, 121102

Murase, K., Inoue, S., \& Nagataki, S. 2008, ApJ, 689, L105

Murase, K., Ahlers, M., \& Lacki, B. C. 2013, Phys. Rev. D, 88, 121301

Murase, K., Inoue, Y., \& Dermer, C. D. 2014, Phys. Rev. D, 90, 023007

Murray, S. G., Power, C., \& Robotham, A. S. G. 2013, Astron. Comput., 3, 23

Norman, C. A., Melrose, D. B., \& Achterberg, A. 1995, ApJ, 454, 60

Padovani, P., \& Resconi, E. 2014, MNRAS, 443, 474

Pfrommer, C. 2008, MNRAS, 385, 1242

Pfrommer, C., \& Enßlin, T. A. 2004, A\&A, 413, 17

Pfrommer, C., Enßlin, T. A., \& Springel, V. 2008, MNRAS, 385, 1211

Pinzke, A., \& Pfrommer, C. 2010, MNRAS, 409, 449

Pinzke, A., Pfrommer, C., \& Bergström, L. 2011, Phys. Rev. D, 84, 123509

Planck Collaboration XVI. 2014, A\&A, 571, A16

Prokhorov, D. A., \& Churazov, E. M. 2014, A\&A, 567, A93

Razzaque, S. 2013, Phys. Rev. D, 88, 081302

Reiprich, T. H., \& Böhringer, H. 2002, ApJ, 567, 716

Riebe, K., Partl, A. M., Enke, H., et al. 2013, Astron. Nachr., 334, 691

Senno, N., Mészáros, P., Murase, K., Baerwald, P., \& Rees, M. J. 2015, ApJ, in press [arXiv: 1501.04934]

Siegal-Gaskins, J. M., Reesman, R., Pavlidou, V., Profumo, S., \& Walker, T. P. 2011, MNRAS, 415, 1074

Sreekumar, P., Bertsch, D. L., Dingus, B. L., et al. 1998, ApJ, 494, 523

Stecker, F. W. 2013, Phys. Rev. D, 88, 047301

Stecker, F. W., \& Venters, T. M. 2011, ApJ, 736, 40

Strong, A. W., Moskalenko, I. V., \& Reimer, O. 2004, ApJ, 613, 956

Sun, M., Voit, G. M., Donahue, M., et al. 2009, ApJ, 693, 1142

Tamborra, I., Ando, S., \& Murase, K. 2014, J. Cosmol. Astro-Part. Phys., 9, 43

Tavecchio, F., \& Ghisellini, G. 2014, MNRAS, submitted [arXiv: 1411.2783]

Taylor, A. M., Gabici, S., \& Aharonian, F. 2014, Phys. Rev. D, 89, 103003

Tinker, J., Kravtsov, A. V., Klypin, A., et al. 2008, ApJ, 688, 709

Tinker, J. L., Robertson, B. E., Kravtsov, A. V., et al. 2010, ApJ, 724, 878

Vannoni, G., Aharonian, F. A., Gabici, S., Kelner, S. R., \& Prosekin, A. 2011, A\&A, 536, A56

Vazza, F., \& Brüggen, M. 2014, MNRAS, 437, 2291

Venturi, T., Giacintucci, S., Brunetti, G., et al. 2007, A\&A, 463, 937

Venturi, T., Giacintucci, S., Dallacasa, D., et al. 2008, A\&A, 484, 327

VERITAS Collaboration. 2012, ApJ, 757, 123

Vogt, C., \& Enßlin, T. A. 2005, A\&A, 434, 67

Voit, G. M. 2005, Rev. Mod. Phys., 77, 207

Völk, H. J., Aharonian, F. A., \& Breitschwerdt, D. 1996, Space Sci. Rev., 75, 279

Waxman, E. 2013, Proc. 9th Rencontres du Vietnam: Windows on the universe,

Aug. 11-17, Quy Nhon, Binh Dinh, Vietnam [arXiv: 1312.0558]

Waxman, E., \& Bahcall, J. 1997, Phys. Rev. Lett., 78, 2292

Waxman, E., \& Bahcall, J. 1999, Phys. Rev. D, 59, 023002

Wiener, J., Oh, S. P., \& Guo, F. 2013, MNRAS, 434, 2209

Winter, W. 2013, Phys. Rev. D, 88, 083007

Winter, W. 2014, Phys. Rev. D, 90, 103003

Zandanel, F., \& Ando, S. 2014, MNRAS, 440, 663

Zandanel, F., Pfrommer, C., \& Prada, F. 2014a, MNRAS, 438, 116

Zandanel, F., Pfrommer, C., \& Prada, F. 2014b, MNRAS, 438, 124

Zatsepin, G. T., \& Kuz'min, V. A. 1966, Sov. J. Exp. Theoret. Phys. Lett., 4, 78 